\title{
Unveiling the oldest and most massive galaxies at very high redshift ${ }^{\star}$
}

\author{
G. Rodighiero ${ }^{1}$, A. Cimatti ${ }^{2}$, A. Franceschini ${ }^{1}$, M. Brusa ${ }^{3}$, J. Fritz ${ }^{1}$, and M. Bolzonella ${ }^{4}$
}

\author{
1 Dipartimento di Astronomia, Università di Padova, Vicolo Osservatorio 2, 35122 Padova, Italy \\ e-mail: giulia.rodighiero@unipd.it \\ 2 Dipartimento di Astronomia, Università di Bologna, via Ranzani 1, 40127 Bologna, Italy \\ 3 Max Planck Institut für Extraterrestrische Physik, Postfach 1312, 85741 Garching bei München, Germany \\ 4 INAF - Bologna, via Ranzani, 40127 Bologna, Italy
}

Received 3 October 2006 / Accepted 5 March 2007

\section{ABSTRACT}

\begin{abstract}
Context. The identification and characterisation of massive galaxies over a wide redshift range allow us to place stringent constraints on the cosmic history of galaxy mass assembly and on current models of galaxy formation and evolution.

Aims. This work explores the existence of high-redshift massive galaxies unveiled with Spitzer+IRAC, but missed by conventional selection techniques based on optical and near-infrared observations.

Methods. To this end, we used multi-wavelength imaging data available for the GOODS-South field $\left(130 \operatorname{arcmin}^{2}\right)$ and selected a flux-limited sample from the IRAC $3.6 \mu \mathrm{m}$ image to $S_{3.6} \geq 1.8 \mu \mathrm{Jy}(m(\mathrm{AB})<23.26)$. In order to identify the most extreme objects and to complement previously published selections in this field, we confined our study to the galaxies undetected by the optical HST+ACS imaging and close to the detection limit of the $K$-band image $(K>23.5 \mathrm{AB})$. Our selection unveiled 20 galaxies on which we performed a detailed analysis.

For each galaxy, we built a spectral energy distribution (SED) based on optical-to- $8 \mu \mathrm{m}$ photometry. The SEDs were then used to estimate the photometric redshifts and to derive the main galaxies' physical properties. Further constraints were also obtained from the available X-ray and $24 \mu \mathrm{m}$ data.

Results. The majority of the sample (14 out of 20) sources show degenerate/bimodal solutions for the photometric redshifts. These can either be heavily dust-enshrouded $\left(A_{\mathrm{V}} \sim 2-4\right)$ starbursts at $2<z<3$ with bolometric luminosities $L_{\mathrm{IR}}>10^{12} L_{\odot}$, or massive post-starburst galaxies in the redshift interval $4<z<9$ with stellar masses of $\sim 10^{11} M_{\odot}$. The remaining six galaxies present a less ambiguous photometric redshift: with the exception of one low- $z$ dusty source, these latter objects favour a low-extinction solution, with four of them showing best-fit photo- $z$ solutions at $z \sim 4$. One galaxy, ID-6, the only source in our sample with both an X-ray and a $24 \mu \mathrm{m}$ detection, might be an extremely massive object at $z \sim 8$ detected during a post-starburst phase with concomitant QSO activity responsible for the $24 \mu \mathrm{m}$ and X-ray emissions (although a lower- $z$ solution is not excluded).

Conclusions. Our investigation of Spitzer-selected galaxies that have very red SEDs and completely undetected in the optical reveals a potential population of massive galaxies at $z \geq 4$, which appear to include significant AGN emissions. These sources may be the oldest stellar systems at $z \sim 4$, given that the estimated ages are close to the age of the Universe at that redshift. We found that these, previously unrecognised, optically obscured objects might provide an important contribution to the massive end $\left(M>10^{11} M_{\odot}\right)$ of the high- $z$ stellar mass function, and they would almost double it. Our evidence in these mature high- $z$ galaxies of the widespread presence of hidden AGNs may have important implications for galaxy formation, due to their feedback effects on the surrounding ISM.
\end{abstract}

Key words. galaxies: high-redshift - galaxies: formation - cosmology: observations - infrared: galaxies

\section{Introduction}

Early attempts to estimate the luminosity and mass functions of galaxies have revealed a surprisingly low rate of evolution of the stellar mass between the present epoch and redshifts $\sim 1$, and particularly so for the most massive systems (Fontana et al. 2004; Bundy et al. 2005; Franceschini et al. 2006). Deep extensive near-IR surveys have recently confirmed the presence of a numerous population of already massive galaxies at $z \sim 2-4$ (e.g., Franx et al. 2003; van Dokkum et al. 2003; Cimatti et al. 2004; Daddi et al. 2004; Yan et al. 2004; Le Fevre et al. 2005; Papovich et al. 2006).

However, very little is currently known about the existence, number density, and properties of massive galaxies

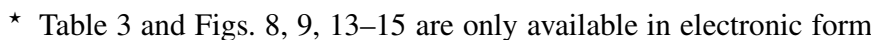
at http://www . aanda.org beyond $z \sim 4$. Mobasher et al. (2005) have recently reported the discovery of a very massive $\left(M>10^{11} M_{\odot}\right)$ galaxy in the GOODS-South field at $z \simeq 6.5$, although a lower- $z$ solution has been suggested by Dunlop et al. (2006). Using the Early Data Release (EDR) by the Ultra Deep Survey (UDS) component of the UKIRT Infrared Deep Sky Survey (UKIDSS), McLure et al. (2006) find nine Lyman-break galaxy candidates at $z \sim 5-6$ with stellar masses higher than $5 \times 10^{10} M_{\odot}$. The formation epochs of such massive galaxies, and of their stellar population contents, cover a wide range of redshifts, whose upper boundary extends well into the reionization epoch (Panagia et al. 2005).

A complementary information is offered by very highredshift quasars (Fan et al. 2003; Fan et al. 2004) located in highly metal-enriched interstellar environments (e.g. Dietrich et al. 2003; Freudling et al. 2003). These cases are a clear indication of already advanced evolutionary stages of the host 
galaxies at such high redshifts (e.g. Maiolino et al. 2006, and references therein), particularly considering that the identified atomic species are subject to delayed supernova enrichment, requiring activity at very high redshifts. More information also comes also from the detection of thermal dust continuum and molecular emissions in these quasars including the farthest known QSO at $z=6.42$, SDSS J1148+5152, indicating the presence of large amounts of dust $\left(M>10^{8} M_{\odot}\right)$ and molecular gas $\left(M>10^{10} M_{\odot}\right.$ ) in their circumnuclear environments (Omont et al. 1996; Omont et al. 2003; Bertoldi et al. 2003; Robson et al. 2004). The formation of dust grains requires condensation processes in addition to the stellar activity required to produce the basic elements $(\mathrm{C}, \mathrm{Si})$, and in normal conditions this occurs in the expanding envelopes of evolved stars (AGB, late giants, with evolutionary timescales of $>10^{8} \mathrm{yr}$ ). If the dust heating came from starburst activity, as has often been suggested, that would imply an enormous star formation rate (SFR) of several hundred to several thousand solar masses per year in the objects.

Finally, the nuclear black-hole mass estimates of the highest$z$ SDSS quasars range from several times $10^{8}$ to several times $10^{9} M_{\odot}$ (e.g. Fan 2006), such that they should have started to form at $z>10$ even with continuous Eddington-limited accretion. Although direct dynamical estimates may suggest a kind of break in the relation between the black hole and the host galaxy mass at the highest redshifts (Walter et al. 2004; Peng et al. 2006), it is hard to imagine that such supermassive black holes do not reside in some kind of forming massive galactic bulge.

In conclusion, both directly detected high- $z$ galaxies and studies of the metal-enriched circumnuclear media in quasars imply enhanced star formation activity at very high redshifts $(z>6)$ in some specific cosmic sites. Establishing how frequent the latter are in representative cosmic volumes would set important constraints on galaxy formation models, particularly considering the fast decline of the dark-matter halo mass function with increasing redshifts. Indeed, the number densities and mass functions of host galaxies at the highest redshifts are far from settled at the moment, while limited information is available only on the most luminous quasars (e.g. from SDSS \& 2DF).

High redshift galaxies are currently selected using a variety of techniques. A very successful approach is based on the detection of the spectral break in the UV continuum blueward of the $\operatorname{Ly} \alpha$ due to the intervening $\operatorname{Ly} \alpha$-forest. By construction, this technique is biased towards star-forming galaxies with restframe UV fluxes that are not strongly reddened by dust extinction (e.g. Steidel et al. 2003; Bouwens et al. 2006). This selection technique is biased against selecting high-redshift galaxies with red spectra, either due to dust extinction or to the presence of mature stellar populations. Thus, other approaches have been recently used to select high- $z$ galaxies in a manner less affected by biases. A successful example is represented by near-IR surveys with or without additional colour or photometric redshift selections (e.g. Cimatti et al. 2002; Franx et al. 2003; Abraham et al. 2004; Daddi et al. 2004), or pure flux-limited optically-selected samples with no colour cuts (e.g. Le Fevre et al. 2005), or submillimetre/millimetre selection of dusty galaxies (Smail et al. 2002; Dannerbauer et al. 2004).

The main question is then whether there are high redshift galaxies that are missed by the current optical and near-IR selection techniques. The advent of the Spitzer Space Telescope opened a new possibility in this respect, as it allows the selection of samples in a spectral region $(3-8 \mu \mathrm{m})$ that was not accessible from the ground. Selection of samples at these wavelengths becomes particularly important for the specific case of high redshift massive galaxies because the $3-8 \mu \mathrm{m}$ selection allows sampling of the rest-frame near-IR of the high- $z$ galaxy spectral energy distributions (SEDs) and is therefore more sensitive to their stellar mass than to their star formation activity, and also much less affected by dust extinction effects.

In this work, we explored whether there are high redshift massive galaxies missed by the conventional selection techniques, but that can be unveiled with Spitzer. For this purpose, we used the multi-wavelength deep-imaging data available for the GOODS-South field (130 $\operatorname{arcmin}^{2}$, Giavalisco et al. 2004) and searched for extreme galaxies in complementary way to the other selections applied in previous surveys in the same field.

The paper is organised as follows. In Sect. 2 we present the multi-wavelength photometric data-set and our sample selection criteria. In Sect. 3 we discuss the model fitting to the observed SEDs, while in Sect. 4 we analyze the infrared colours of our sample. Section 5 illustrates the X-ray properties of the sample sources. In Sect. 6 we report the detection of a candidate massive galaxy at $z \sim 8$. Section 7 presents a general discussion of the statistical properties of our sample. Finally, in Sect. 8 we summarise the main results of this paper. We adopt $\Omega_{\mathrm{m}}=0.3$, $\Omega_{\Lambda}=0.7$, and $H_{0}=70 \mathrm{~km} \mathrm{~s}^{-1} \mathrm{Mpc}^{-1}$ for the cosmological parameters. All magnitudes are given in the $\mathrm{AB}$ system.

\section{Observations and sample selection}

With the aim of studying the multiwavelength photometry of extragalactic sources measured by different instruments, we need to measure the bulk of the emission from each object in each photometric band. Only with this kind of approach can an SED be considered reliable. In our work, we performed aperture photometry in each band.

\subsection{Deep Spitzer near- and mid-IR photometric imaging}

As part of the GOODS project, the Spitzer Space Telescope has recently surveyed the CDFS field in the IR between 3.6 and $8.0 \mu \mathrm{m}$ using IRAC and in the range $24-160 \mu \mathrm{m}$ using MIPS. The fully reduced data were publicly released by the GOODS team and are available through the World Wide Web ${ }^{1}$.

In this paper we exploit a galaxy catalogue that we derived from the $3.6 \mu \mathrm{m}$ IRAC public raw data and recently used to derive the luminosity and mass function by morphological type (Franceschini et al. 2006). In order to obtain the most accurate SEDs, we recomputed the fluxes of each source by performing aperture photometry in the four IRAC bands at the positions originally detected in the IRAC $3.6 \mu \mathrm{m}$ channel. Assuming that essentially all the sample sources are seen as point-like by the IRAC $\sim 2$ arcsec FWHM PSF imager, we used SExtractor (Bertin \& Arnouts 1996) to compute the fluxes within a 3.8 arcsec diameter aperture. This choice is supported by an accurate analysis performed by the SWIRE team ${ }^{2}$. They constructed colour-magnitude diagrams for various types of objects, in particular main-sequence stars. It was then found that the scatter in these diagrams is minimised through the use of a 3.8 diameter aperture and corresponds to roughly twice the beamwidth. To obtain total fluxes, we then applied the correction factors indicated by the SWIRE team ${ }^{3}$. We independently

\footnotetext{
1 http://data.spitzer.caltech.edu/popular/goods

2 http://data.spitzer.caltech.edu/popular/swire/ 20050603_enhanced_v1/

3 See Note 2.
} 
verified that the IRAC/SWIRE aperture corrections are consistent with those derived by fitting the radial brightness profiles of stars in the GOODS fields. In the case of extended sources, we used Kron-like magnitudes (AUTO_MAG output parameter in SExtractor). Our sample turned out to be $\sim 60 \%$ complete above $1 \mu \mathrm{Jy}\left(m_{3.6}=23.9\right), \sim 75 \%$ complete above $2 \mu \mathrm{Jy}\left(m_{3.6}=23.15\right)$, $\sim 90 \%$ at $5 \mu \mathrm{Jy}\left(m_{3.6}=22.15\right)$, and more than $\sim 95 \%$ above $10 \mu \mathrm{Jy}\left(m_{3.6}=21.4\right)$.

The MIPS public dataset includes calibrated maps and a catalogue of $24 \mu \mathrm{m}$ sources with flux densities $S_{24}>80 \mu \mathrm{Jy}$. The photometry is based on a PSF fitting algorithm, where the SExtractor positions of the IRAC sources are used as input to the MIPS source extraction process. The MIPS $24 \mu \mathrm{m}$ PSF was generated from isolated sources in the image, and renormalised based on the aperture corrections published in the MIPS Data Handbook (v2.1, Sect. 3.7.5, Table 3.12).

To extend the $24 \mu \mathrm{m}$ sample to fainter fluxes, we ran an independent PSF fitting algorithm that we had already successfully applied in the GOODS-xFLS/EN1 science verification field (Rodighiero et al. 2006). By these means we have extended the $24 \mu \mathrm{m}$ sample down to $S_{24}>20 \mu \mathrm{Jy}$.

\subsection{Near-IR ground-based imaging}

As part of GOODS, near-infrared imaging observations of the CDFS were carried out in the $J, H$, and $K_{\mathrm{s}}$ bands, using the ISAAC instrument mounted on the ESO VLT. We made use of the publicly available $J, H$ and $K_{\mathrm{s}}$ imaging (version 1.0, released $^{4}$ by the ESO/GOODS team in April 2004). This data release includes 21 fully-reduced VLT/ISAAC fields in $J, H$, and $K_{\mathrm{s}}$, covering $130 \mathrm{arcmin}^{2}$ of the GOODS/CDFS region. It also includes mosaics of the coadded tiles as single FITS files in $J$ and $K_{\mathrm{s}}$ bands, as well as the corresponding weight maps. To provide a homogeneous photometric calibration across the entire field, all images were rescaled to the same zero point (26.0). The final mosaics have a pixel scale of $0.15^{\prime \prime}$.

We measured the $J, H$ and $K$ band magnitudes with SExtractor at the positions of the $3.6 \mu \mathrm{m}$ IRAC selected sources through circular apertures with diameters of 2 arcsec. Mobasher et al. (2005) find that the photometric curve of growth converges at this aperture, which represents the best compromise between convergence of the total flux and the effects of systematic uncertainty in the background subtraction.

For undetected ISAAC sources, we initially computed the upper limits to the flux by measuring the signal in 400 random sky positions. We used 2-arcsec aperture diameters and calculate the value of the standard deviation from the distribution of the measured aperture fluxes. We obtained $1 \sigma$ values of $26.12,25.82$, and $25.12 \mathrm{mag}$ for the $J, H$, and $K$ band, respectively. However, given that the depth of the ISAAC imaging varies significantly across the CDFS field, we preferred to compute independent fluxes for each undetected source. Following the approach of Dunlop et al. (2006), we perfomed our own manual photometry through a 2 -arcsec diameter aperture at the IRAC-selected positions.

\subsection{ACS/HST optical imaging}

The core of the GOODS project was the acquisition and data reduction of high-resolution HST/ACS imaging obtained as an HST Treasury Program (Giavalisco et al. 2004). The GOODS ACS/HST Treasury Program has surveyed two separate fields

\footnotetext{
${ }^{4}$ http://www. eso.org/science/goods/releases/20040430/
}

(the CDFS and the Hubble Deep Field North) with four broadband filters: $F 435 W(B), F 606 W(V), F 775 W(i)$ and $F 850 L P(z)$. In August 2003 the GOODS team released version 1.0 of the reduced, stacked, and mosaiced images for all the data acquired over the five epochs of observation. To improve the point-spreadfunction (PSF) sampling, the original images, which had a scale of $0.05 \mathrm{arcsec} / \mathrm{pixel}$, were drizzled on to images with a scale of $0.03 \mathrm{arcsec} / \mathrm{pixel}$.

The dataset is complemented by the ACS/HST catalogues released by the HST/GOODS team in October 2004, containing the photometry in $B, V, i$ and $z$ bands. The source extraction and the photometric measurements were performed by the GOODS team running a modified version of SExtractor on the version 1.0 images. We have considered aperture magnitudes using a 1 arcsec diameter. For undetected IRAC sources, photometry in the ACS $B, V, i$, and $z$ bands was measured as upper limits with the same procedure adopted for the ISAAC imaging. We used 1 arcsec diameter apertures and adopted the $3 \sigma$ value as an upper limit to the flux of undetected sources.

\subsection{Sample selection}

Our main aim is to search for galaxies possibly missed by previous surveys and to find new candidates of high-redshift massive systems. In a recent paper, Dunlop et al. (2006) makes use of a $K$-band selected sample brighter than $K=23.5 \mathrm{AB}$ mag to investigate the high- $z$ galaxy population in the GOODS-S field. They conclude that there is no convincing evidence of any galaxies with $M>3 \times 10^{11} M_{\odot}$ at $z>4$.

In order to search for extreme galaxies "dark" at optical wavelengths and ultrafaint in the near-IR, and to complement the selection applied by Dunlop et al. (2006) in the same field, we selected our sample starting from the Spitzer IRAC $3.6 \mu \mathrm{m}$ image, and applied the following selection criteria:

(1) flux-limited selection from the IRAC $3.6 \mu \mathrm{m}$ image down to $S_{3.6} \geq 1.8 \mu \mathrm{Jy}(m(\mathrm{AB})<23.26)$

(2) objects undetected in any of the optical HST+ACS images at the $1 \sigma$ level (i.e. $z>28.14$ );

(3) objects close to the detection limit of the VLT+ISAAC $K$-band deep image $(K>23.5 \mathrm{AB}$, a complementary criterion to that of Dunlop et al. 2006, including objects with $K<23.5)$.

In order to avoid potential photometric uncertainties from the blending due to the large IRAC PSF, we excluded a few blended sources from our sample. We found twenty sources satisfying the above conditions. This final sample was further investigated on ISAAC images: the twenty sources turned out to all be undetected in the $J$-band (at least at the ISAAC sensitivity), while only two candidates showed a faint counterpart in the $H$-band image. Three sources remain undetected even in the $K$-band ISAAC image.

To illustrate the effects of our selection criteria, we report the $K-[3.6]$ versus $z-[3.6]$ colours for our complete GOODS/CDFS sample with $S_{3.6}>1.8 \mu \mathrm{Jy}$ in Fig. 1 (top panel). For comparison, we also show here the colour-colour plots of five spectral template SEDs: a 10-Gyr old passive elliptical, a star-forming and a post-starburst galaxy ${ }^{5}$, a solar-metallicity SSP with an age of 1 Gyr (unextinguished) and a younger extinguished SSP of

5 The synthetic spectra are taken from the set of template used in Fritz et al. (2007, submitted). The first is taken as representative of a galaxy during a post-starburst phase with a second main episode of star formation, forming $\sim 10 \%$ of the total stellar mass, at $\sim 10^{8}$ years while 


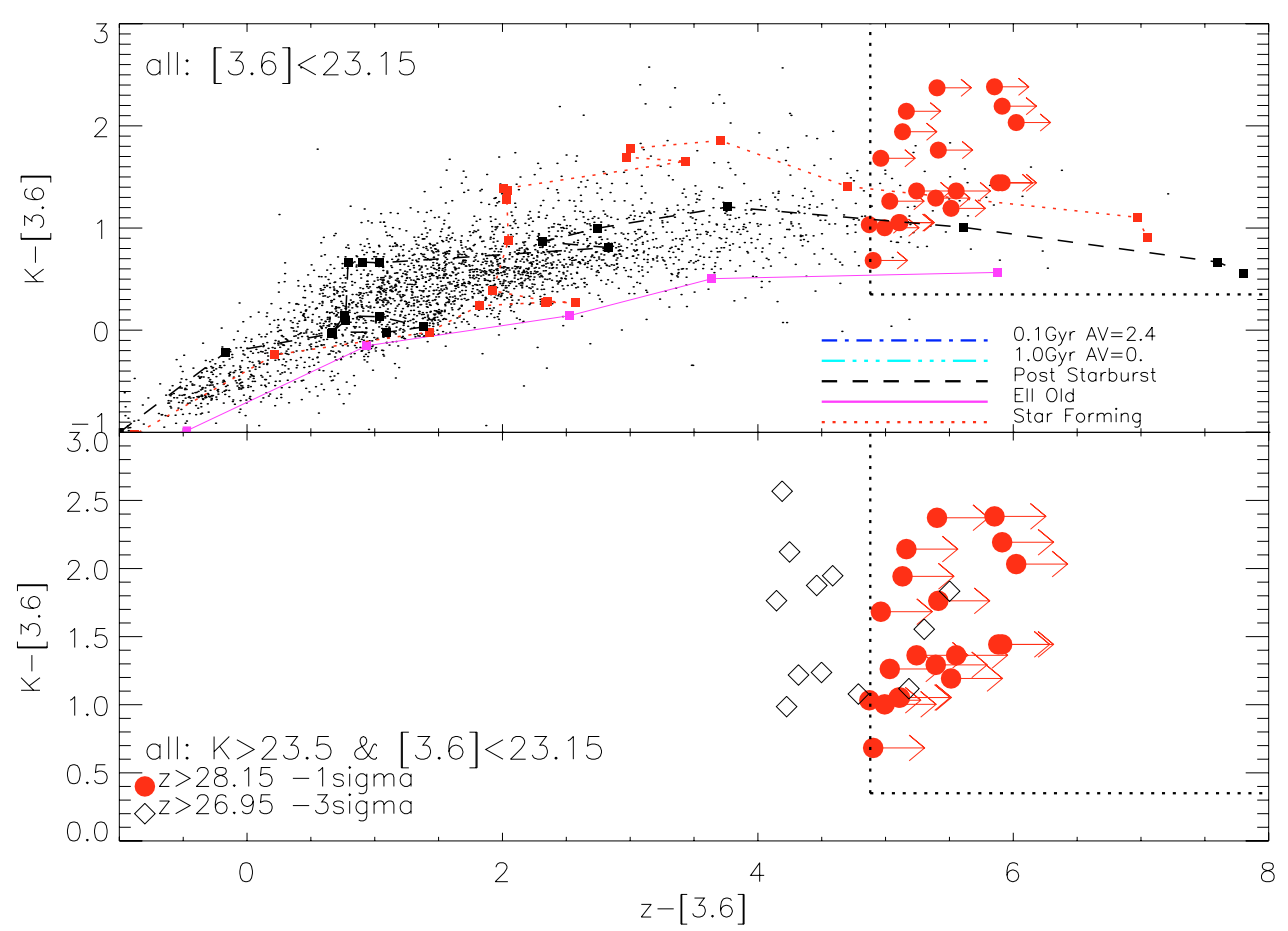

Fig. 1. Plots of the $K-$ [3.6] versus $z-$ [3.6] colours for our reference GOODS/CDFS IRAC-selected sample with $S_{3.6}>1.8 \mu$ Jy (small black dots in the top panel). The objects from our high-redshift sub-sample correspond to the big red circles with $z$-band limits. Data are compared with the colours of five spectral templates: a $10 \mathrm{Gyr}$ old and passive elliptical (solid line), a star-forming (dotted line) and a post-starburst galaxy (dashed), a single simple stellar population (SSP) with an age of $1 \mathrm{Gyr}$ (unextinguished, three-dots-dashed line) and a younger extinguished SSP of $100 \mathrm{Myr}$ $\left(A_{\mathrm{V}}=2.4\right.$, dot-dashed line). The predicted colours are shown as a function of redshift starting from $z=0$ with increasing steps of 0.5 (the $z=0$ values fall typically in the bottom-left part of the panel). The bottom panel zooms onto the colours of our candidate very-high-redshift objects undetected in the $z$-band down to $z=28.15$ ( $1 \sigma$ limit), and an additional sample of objects fainter than $z=26.95$ ( $3 \sigma$ limit).

$100 \mathrm{Myr}\left(A_{\mathrm{V}}=2.4\right)$. The predicted colours are shown as a function of redshift starting from $z=0$ with increasing steps of 0.5 (the $z=0$ value falls in the bottom left part of the panel). The bottom panel zooms onto the colours of our candidate veryhigh-redshift objects undetected in the $z$-band down to $z=28.15$ ( $1 \sigma$ limit), and, for comparison, an additional sample of objects fainter than $z=26.95$ ( $3 \sigma$ limit $)$.

We note that $\sim 40$ sources, falling in the colour-colour region populated by our final sample, escaped our selection criteria being all brighter in the $K$ - and $z$-bands with respect to our imposed constraints $(K>23.5$ and $z>28.14)$.

A summary of the multiwavelength identifications of the $3.6 \mu \mathrm{m}$ objects is presented in Fig. 2. For each source we report here postage stamps of $5^{\prime \prime} \times 5^{\prime \prime}$ size in the ACS $z$-band, $8^{\prime \prime} \times 8^{\prime \prime}$ in the ISAAC and IRAC bands and of $17^{\prime \prime} \times 17^{\prime \prime}$ in the $24 \mu \mathrm{m}$ MIPS band. To look for any possible faint optical detection, we stacked together the images of all sample sources in the four ACS bands. The result of this stacking procedure is presented in Fig. 3, showing the complete absence of optical signal at the position of the selected sources.

The photometric data used to construct the spectral energy distributions discussed in the next section are presented in Table 1. Negative values correspond to upper limits. We adopt a common value of $10 \%$ (15\%) for the measured fluxes as photometric errors for the IRAC (MIPS) bands, in order to reflect the systematic uncertainties of the instruments The main contributions to these uncertainties are due to the colour-dependence in the flat field and to the absolute calibration (see for example

the second is build with a main-impulsive burst at $\sim 13$ Gyr and a continuous star formation that is truncated at $2 \times 10^{7}$ years.
Lacy et al. 2005, and the IRAC and MIPS Data Handbook). For the ISAAC $K$ - and $H$-band fluxes, we adopt as photometric error a standard value of $0.3 \mathrm{mag}$ (as derived from the standard deviation statistics from our set of Monte Carlo simulations discussed in Franceschini et al. 2006). The observational SEDs for all our sample sources are plotted in Figs. 9 and below.

\subsection{Contamination by galactic stars}

We first considered the possibility that some sources within our sample could be misidentified Galactic cool stars.

We verified that ultra-cool galactic stars (like $M$ and L dwarfs) show colours (e.g. $K-[3.6]$, see Fig. 4) that are in general much bluer than those of our sources. Moreover, the peak of the stellar emissions of $\mathrm{M}$ dwarfs falls shortward $(\lambda \sim 1-2 \mu \mathrm{m})$ than observed in the SEDs of our sample ${ }^{6}$.

Another possible contaminant is represented by evolved dusty stars, like AGBs (in particular carbon stars). The spectra of these objects should reproduce the SEDs of our sample in the $0.4-8.0 \mu \mathrm{m}$ interval range (see Fig. 4). However, the detection of several AGB stars in an area of only $130 \mathrm{armin}^{2}$ at the magnitude limit of $K<23.5$ is very much in excess of what is e.g. observed in the LMC (Cioni et al. 2006). In addition, these objects are so bright that they should be moved far away from our Galaxy (at least $\sim 15 \mathrm{Mpc}$ ) in order to match the observed fluxes of our sample objects.

${ }^{6}$ We made use of the colour predictions from the stellar model of Jarrett et al. (1994). We also used the spectral templates of the ISO standard stars available at http://www.iso.vilspa.esa.es/users/expl_lib/ISO/wwwcal/ 


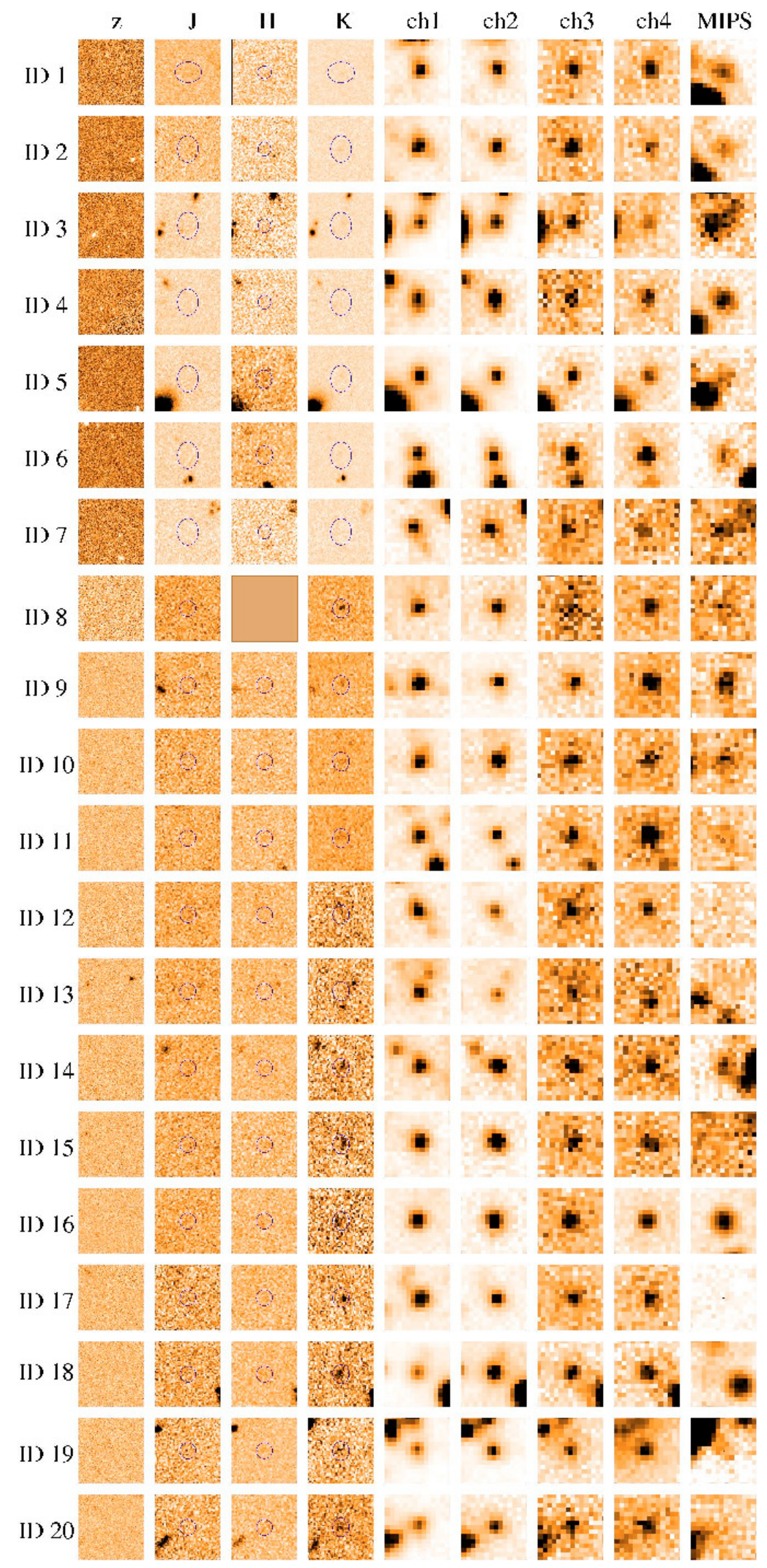

Fig. 2. Multiwavelength identification of the twenty $3.6 \mu \mathrm{m}$-selected objects. For each source we report a postage of $5^{\prime \prime} \times 5^{\prime \prime}$ in the ACS $z$-band, of $8^{\prime \prime} \times 8^{\prime \prime}$ in the ISAAC and IRAC bands and of $17^{\prime \prime} \times 17^{\prime \prime}$ in the $24 \mu \mathrm{m}$ MIPS band. North is up, East is to the left. 


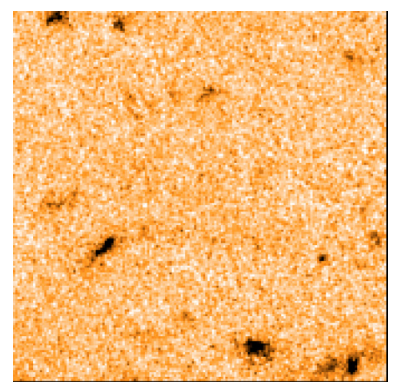

Fig. 3. Postage stamps showing the result of the stacking procedure of all ACS bands $(B, V, i, z)$ at the positions of the twenty near-IR dark sources. No optical signal is detected. The postage has a size of $5^{\prime \prime} \times 5^{\prime \prime}$.

Young stellar objects (YSOs) may also be considered. Although their spectra resemble those of our sources, they are usually associated with extended molecolar clouds in Galactic star-forming regions that are obviously absent in the GOODS-South field.

An interesting possibility is the potential contamination by Galactic brown dwarfs. Mannucci et al. (2006) have recently suggested that a pair of faint $z$-dropout sources $(z-J>0.9)$ in the CDFS are compatible with the expected colours of brown dwarfs. We checked this hypothesis in Fig. 5, where we report the $J-K$ colour versus the $K-$ [4.5] colour for our candidate high-redshift galaxies. The $J$-band magnitudes appearing in this plot correspond to our $2 \sigma$ value $(J=26.29)$. The dashed region indicates the expected colours for galactic brown dwarfs (adapted from Mannucci et al. 2006, that used the stellar models of Allard et al. 2001). Clearly, all sources in our sample present $J-K$ colours that are much redder than expected for brown dwarfs.

\section{Infrared colours}

\subsection{Near-IR to IRAC colours}

To start constraining the nature of our selected galaxies, we first compared their mid-IR properties with recently suggested colour-colour diagnostic plots. While the composite spectra of the stellar populations in normal galaxies produce SEDs peaking at approximately $1.6 \mu \mathrm{m}$, the UV to mid-IR continua of AGNs are dominated by power-law emission. Based on this, Lacy et al. (2004) used IRAC colours from the Spitzer First Look Survey to identify AGNs. A region in the [4.5]-[8.0] versus [3.6]-[5.8] colour plot where AGN are expected to lie is shown in Fig. 6.

For comparison to the twenty near-IR dark objects, we also report the colour distribution of the GOODS/MUSIC sample (Grazian et al. 2006) with $S(8 \mu \mathrm{m})>1 \mu \mathrm{Jy}$. The colour tracks for a dusty starburst (Arp 220), a Seyfert 1 galaxy, and a passive elliptical are also shown as a function of redshift, with an increasing step size of 0.5 , starting from $z=0.5$ (as marked in the plot for each template). All sources fall within the AGN area. However, this is not necessarily an indication of an AGN dominance in our sample. Indeed, Fig. 6 also shows the colour distribution of a sample of SCUBA radio-selected sources detected at Spitzer wavelengths (Frayer et al. 2004), and the SWIRE/Chandra sample selected in the EN1 field by Franceschini et al. (2005). Clearly, both star-forming galaxies, ellipticals, and AGNs at $z>2$ can all reproduce the IRAC colours of our sample, showing a limited diagnostic power by the test.

\subsection{Near-IR - to - mid-IR colours}

It is remarkable that half of our $3.6 \mu \mathrm{m}$ selected sources in our sample (11 out of 20) reveal significant $24 \mu \mathrm{m}$ excess. Given the MIPS and IRAC limiting fluxes, this is inconsistent with a purely passive galaxy at any redshift, and clearly indicates some activity taking place in the objects. The nature of this activity (star formation, AGN, or the concomitance of both at the same time) will be the subject of our investigation here.

In Fig. 7 the colour [8]-[24] is shown as a function of the colour $K-$ [8] (see also Webb et al. 2006). We also report here colour tracks for starbursts galaxies (Arp 220), AGNs (Seyfert 1 and Seyfert 2), a combined quasar/ultraluminous infrared galaxy (ULIRG) sources like the type-1 QSO Mrk 231 the type-2 quasar/ULIRG Superantennae (IRAS 19254s; Berta et al. 2003), and a passive elliptical. These templates are derived from the spectral library reported in Polletta et al. 2006 (see also Franceschini et al. 2005). The predicted colours are shown as a function of redshift with increasing step size of 0.5 , starting from $z=0.5$ (as marked in the plot for each template).

The extremely red infrared colours of our sample are difficult to explain. The $K-[8]$ colour on the $\mathrm{X}$-axis might be consistent with the SED of a starburst galaxy, like Arp 220, or alternatively with a passively evolving galaxy, both at $z>3$. Lower-redshift solutions require additional extinction $\left(A_{\mathrm{V}} \sim 2\right)$ to that of the Arp 220 spectrum. The very red [8]-[24] ratio may be consistent alternatively with a moderate-redshift $(z<3)$ dusty starburst (Arp 220) or with a dusty quasar.

The combined set of colours are reproduced only by high redshift $(z>2.5)$ ULIRGs with concomitant QSO activity (the type-2 quasar Superantennae, more marginally by the infraredluminous type-1 QSO Mrk231). This colour-colour plot of Fig. 7 will be considered in later applications.

\section{Spectral energy distribution analysis}

\subsection{Synthetic spectral models and photometric redshift estimates}

We made use of the Hyperz code (Bolzonella et al. 2000) to estimate the photometric redshifts of each source. The whole broadband photometric dataset available to us was exploited, with the exclusion of the $24 \mu \mathrm{m}$ flux (the stellar-population synthesismodel template spectra do not include dust emission). The fitting procedure was based on a maximum-likelihood algorithm and the quality of the fit is investigated by means of $\chi^{2}$ statistics. The code computes the $\chi^{2}$ for a given number of templates, which differ for star formation histories, metallicities and ages, and finds the best-fitting template among them.

We used two stellar-population synthesis models to fit the observed SEDs: the Bruzual \& Charlot (hereafter BC03) and the Maraston (2005, hereafter MA05) models. In both cases, we assumed exponentially decreasing star-formation rates (SFR) parameterized by a time scale $\tau$. We considered the following set of values for $\tau$ : $0.1,0.3,1,2,3,5,15,30$ Gyr. Moreover, we included a case with constant SFR and a single-burst model corresponding to an individual simple stellar population (SSP). For the BC03 model we used a Chabrier initial mass function (IMF). The MA05 evolutionary tracks available to us have been generated with a Salpeter IMF. To convert mass estimates obtained with Salpeter IMF to Chabrier IMF, we used a constant value of 0.23 dex (which corresponds to correct the masses computed with Salpeter IMF downwards by a factor 1.7 ). This value was obtained by comparing the stellar masses estimated with 
Table 1. Photometric properties of the sample objects.

\begin{tabular}{|c|c|c|c|c|c|c|c|c|c|c|c|c|c|c|}
\hline D & $\overline{\overline{\mathrm{RA}}}$ & Dec & $b$ & 0 & $i$ & $z$ & $J$ & $\overline{\bar{H}}$ & $\overline{\bar{K}}$ & {$[3$.} & {$[4$.} & {$[5$.} & [8.0] & [24] \\
\hline & J2000 & $\mathrm{J} 2000$ & mag & mag & mag & mag & mag & mag & mag & mag & mag & $\mathrm{mag}$ & $\mathrm{mag}$ & $\mathrm{mag}$ \\
\hline 1 & 53.12440 & -27.88268 & -27.61 & -27.72 & -27.15 & -26.94 & -25.41 & -25.65 & 24.49 & 22.72 & 22.35 & 22.13 & 21.62 & 18.69 \\
\hline 2 & 53.22620 & -27.85910 & -27.61 & -27.72 & -27.15 & -26.94 & -26.19 & -24.97 & 23.95 & 22.58 & 22.32 & 21.92 & 22.20 & 19.03 \\
\hline 3 & 53.06607 & -27.83178 & -27.61 & -27.72 & -27.15 & -26.94 & -25.78 & -25.17 & 23.67 & 22.22 & 22.06 & 1.94 & 22.08 & 19.27 \\
\hline 4 & 53.19752 & -27.81387 & -27.61 & -27.72 & -27.15 & -26.94 & -26.86 & -25.06 & -24.86 & 23.17 & 22.85 & 2.83 & 21.82 & 18.78 \\
\hline 5 & 146 & -27.81118 & -27.61 & -27.72 & -27.15 & -26.94 & -25.45 & 24.94 & 24.15 & 22.11 & 1.81 & 1.60 & .37 & 19.63 \\
\hline 6 & 5 & -27 & -27.61 & -27.72 & -27.15 & -26.94 & -25.65 & 24.68 & 24.42 & 2.22 & 5 & 2 & .85 & 19.27 \\
\hline 7 & & & -2 & & -27 & -26. & .37 & 25.06 & 4.30 & 23.26 & 3.02 & & 2.93 & 20.70 \\
\hline 8 & 5 & -2 & -27 & -27.72 & -27 & -26 & -26.27 & _* & 23.92 & 23.23 & 22.86 & 2.64 & 21.87 & 00.00 \\
\hline 9 & & 0 & -27 & -2 & -2 & -26 & -26.65 & -25.92 & 2. & 22.73 & 22.13 & 1.52 & 1.46 & 19.69 \\
\hline 10 & & -2 & -2 & 72 & -27 & -26 & -25 & -25 & -25 & .97 & 54 & 42 & 22.33 & 19.72 \\
\hline 11 & & -2 & -2 & -2 & -2 & -26 & -2 & -26 & 24 & 23.03 & 7 & 99 & 14 & 00.00 \\
\hline 12 & 5 & -27 & -27.61 & -27.72 & -27.15 & -26.94 & -25.39 & -25.20 & -24.95 & 23.00 & 22.57 & 2.26 & 21.85 & 00.00 \\
\hline 13 & 5 & -27 & -27.61 & -27.72 & -27.15 & -26.94 & -26.79 & -26.02 & 24.15 & 23.14 & 22.82 & 2.68 & 22.64 & 00.00 \\
\hline 14 & 53 & -27.84421 & -27.61 & -27.72 & -27.15 & -26.94 & -25.55 & -24.88 & 24.37 & 23.10 & 22.76 & 22.51 & 22.35 & 19.40 \\
\hline 15 & 12 & -27.82529 & -27.61 & -27.72 & -27.15 & -26.94 & -26.05 & -25.08 & 24.26 & 22.89 & 22.56 & 22.35 & 22.39 & 00.00 \\
\hline 16 & 94 & -27.81414 & -27.61 & -27.72 & -27.15 & -26.94 & -26.01 & -24.82 & -24.08 & 23.02 & 22.72 & 22.10 & 20.84 & 17.78 \\
\hline 17 & 01 & -27.80455 & -27.61 & -27.72 & -27.15 & -26.94 & -25.54 & -25.38 & 24.04 & 22.74 & 22.55 & 22.39 & 22.46 & 00.00 \\
\hline 18 & 47 & -27.77972 & -27.61 & -27.72 & -27.15 & -26.94 & -25.54 & -24.91 & 23.70 & 22.25 & 22.10 & 21.80 & 21.86 & 00.00 \\
\hline 19 & 983 & -27.74309 & -27.61 & -27.72 & -27.15 & -26.94 & -26.25 & -26.02 & 24.67 & 22.28 & 21.80 & 1.32 & 20.50 & 00.00 \\
\hline 20 & 53.12762 & -27.70684 & -27.61 & -27.72 & -27.15 & -26.94 & -25.37 & -24.82 & 23.82 & 2.62 & 2.41 & 2.33 & 1.92 & 00.00 \\
\hline
\end{tabular}

* Source \#8 is out of the $H$ band image.

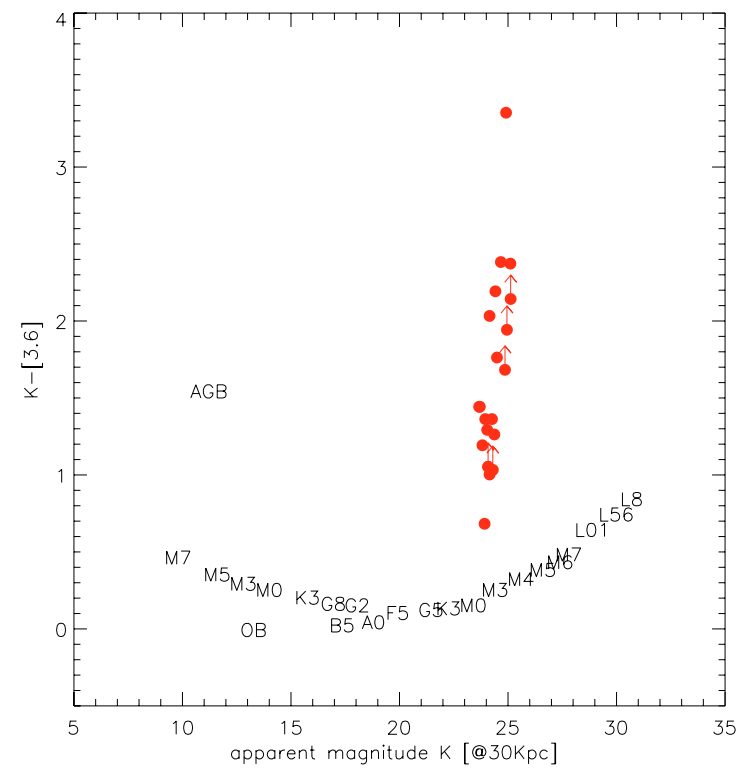

Fig. 4. $K$ - [3.6] colour versus $K$ magnitude plot for our candidate highredshift galaxies (filled circles) and for galactic stars of different spectral types, as indicated. $K$ magnitudes are calculated by setting stars at a $30 \mathrm{kpc}$ distance.

BC03 models built with different IMFs for an observed spectroscopic sample of galaxies. In this way we obtained the mass difference expected for galaxies with the same colours.

The extinction parameter was allowed to span the widest conceivable range of values $0<A_{\mathrm{V}}<10$ (with a step of $\left.d\left(A_{\mathrm{V}}\right)=0.1\right)$. We assumed the extinction law by Calzetti et al. (2000), and the metallicity was set to the solar value, in order to minimize the number of free parameters at play. We also accounted for Lyman-series absorption due to HI clouds in the intergalactic medium, following the prescription of Madau (1995).

Even if the reddening is formally allowed to reach extreme values, we recall that values of $A_{\mathrm{V}}$ exceeding 5-6 mag have been observed only in the inner part (i.e. the central $1-2 \mathrm{kpc}$ )

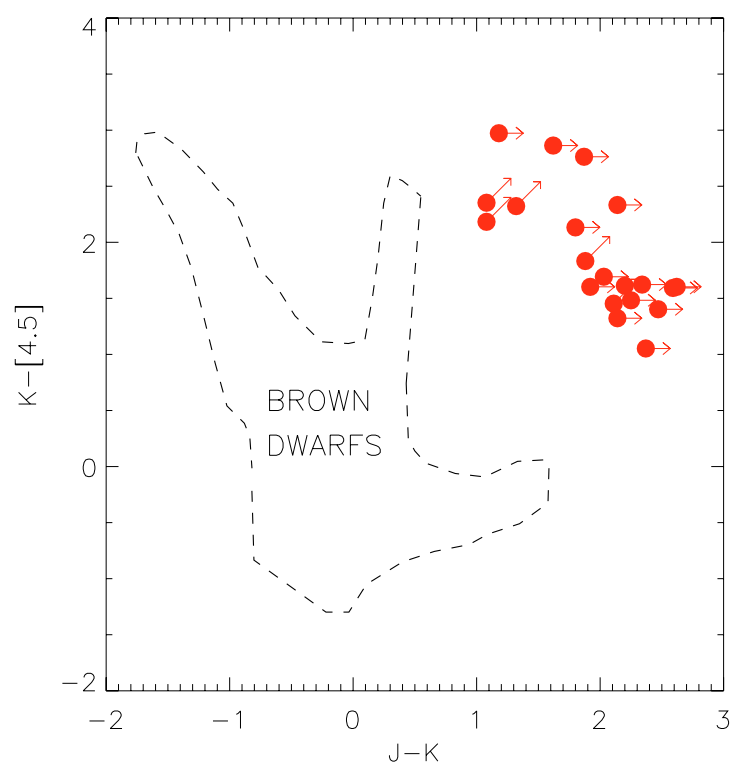

Fig. 5. $J-K$ colour versus $K-$ [4.5] colour for our candidate highredshift galaxies (filled circles). The $J$-band magnitudes adopted in this plot correspond to the $2 \sigma$ value $(J=26.29)$. The dashed region indicates the expected colours for Galactic brown dwarfs, as recently suggested by Mannucci et al. (2006).

of local luminous IR galaxies (e.g. Mayya et al. 2004; Poggianti et al. 2001). In comparison, at high redshift $(2<z<3)$ the dusty submillimeter galaxies have typical average extinction around $A_{\mathrm{V}} \sim 2.4$ (Smail et al. 2004; Knudsen et al. 2005). Another class potentially including heavily obscured sources is that of distant red galaxies (DRGs) and dusty extremely red objects (EROs). However, even in this case the reddening has typical values around $A_{\mathrm{V}}<\sim 3.0$ at $2<z<3$ (Cimatti et al. 2003; Moustakas et al. 2004; Stern et al. 2006; Papovich et al. 2006). A hyper extremely red object (HERO) has been proposed to lie at $z=2.4$ with $A_{\mathrm{V}}=4.5 \mathrm{mag}$ (Im et al. 2002). However, no 

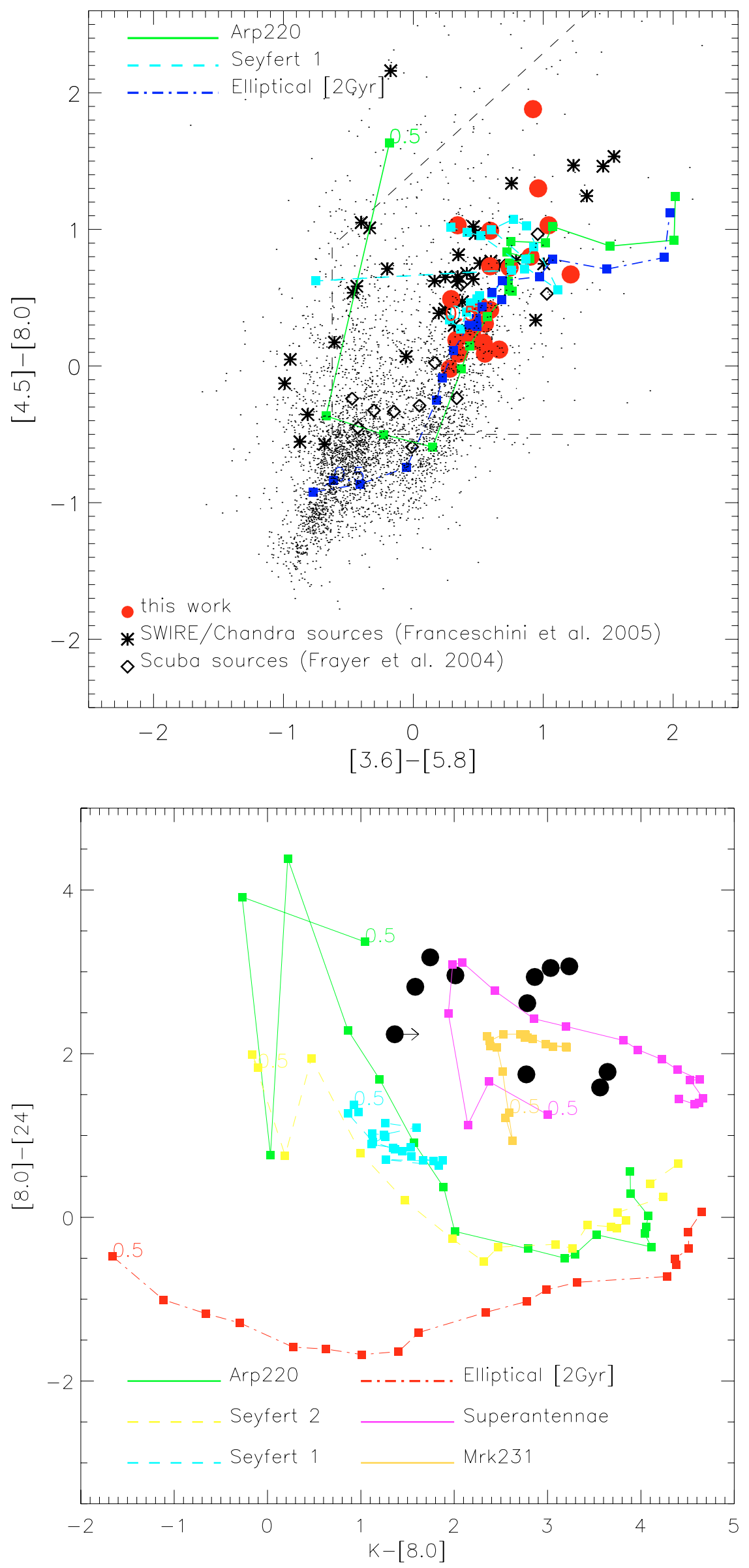

Fig. 6. The IRAC colour [8.0]-[4.5] is shown against the colour [5.8]-[3.6]. The dashed line marks the region dominated by AGNs in the redshift range $1<z<3$ according to Lacy et al. (2005). The near-IR dark objects of our sample are plotted as red filled circles. For comparison, we report a sample of SCUBA radio-selected sources detected at Spitzer wavelengths (open diamonds, Frayer et al. 2004) and a sample of Chandra sources detected in the SWIRE survey by Franceschini et al. (2005). We plot the evolutionary tracks for a reddened starburst (Arp 220 - green solid lines), a Seyfert 1 galaxy (dashed cyan lines), and a passive elliptical (dot-dashed red lines). The predicted colours are shown as a function of redshift with increasing step size of 0.5 , starting from $z=0.5$ (as marked in the plot for each template). For comparison, we also report the colour distribution (dotted points) of the GOODS/MUSIC sample (Grazian et al. 2006) with $S(8 \mu \mathrm{m})>1 \mu \mathrm{Jy}$.

Fig. 7. The colour [24]-[8] is shown as a function of the colour [8]-[2.2]. Our sources are plotted as big filled circles. We also report evolutionary tracks for starbursts galaxies (Arp $220-$ green solid lines), AGNs (Seyfert 1 - dashed cyan lines, Seyfert 2 - dashed yellow line), combined quasar/ultraluminous infrared galaxy (ULIRG) sources like the type-1 QSO Mrk 231 (orange solid lines) and the type-2 quasar/ULIRG Superantennae South (IRAS 19254s; Berta et al. 2003, magenta lines), and a passive elliptical (dotdashed red lines). The predicted colours are shown as a function of redshift with increasing step size of 0.5 , starting from $z=0.5$ (as marked in the plot for each template). 
convincing evidence of galaxies with a global value of $A_{\mathrm{V}}$ exceeding 5-6 mag has been reported until know.

\subsection{Additional constraints from $24 \mu \mathrm{m}$ flux}

To reduce the uncertainties in the extinction estimate, we considered the MIPS $24 \mu \mathrm{m}$ flux in addition to the $0.4-8 \mu \mathrm{m}$ photometric data. The dust-emission spectrum longwards of a few $\mu \mathrm{m}$ (restframe) was modelled by assuming the observed IR SED of Arp 220 and then compared to the flux detected in the MIPS band. The Arp 220 IR template corresponds to the spectrum of a highly absorbed ultra-luminous IR starburst, a conservative choice for our analysis.

The predicted IR emission for each one of our objects is calculated as the difference between the unextinguished and the extinguished optical emission, assuming that all the flux absorbed by dust is re-processed and re-emitted longwards of a few $\mu \mathrm{m}$. The Arp 220 template is then rescaled in such a way that its bolometric emission between 8 and $1000 \mu \mathrm{m}$ coincides with the dustreprocessed luminosity. Finally, the properly scaled Arp 220 SED is K-corrected and convolved with the MIPS filter response to compare with the observed $24 \mu \mathrm{m}$ flux or flux upper limit (see also Berta et al. 2004). With this procedure, we were able to use the measured $24 \mu \mathrm{m}$ flux as a constraint on the maximum $A_{\mathrm{V}}$ allowed to each photometric redshift solution.

If compared to other typical LIRG galaxies (e.g. the prototype local starburst M 82), the ULIRG Arp 220 is characterised by a much more peaked FIR emission and dust self-absorption. The choice of such an extreme galaxy as an IR template then implies much higher bolometric infrared luminosities for a given $24 \mu \mathrm{m}$ flux, providing an indication of the maximun value of $A_{\mathrm{V}}$ consistent with the observed $24 \mu \mathrm{m}$ flux.

The results of the SED fitting analysis are reported in Fig. 8, where for each source we show the confidence levels derived from the $\chi^{2}$ statistics (averaged over all the available free parameters) in the left panel as a function of redshift $z$ and $A_{\mathrm{V}}$, for the BC03 models (the MA05 model provides equivalent results in terms of photometric redshift solutions, see Sect. 4.4). We report the $68 \%, 90 \%, 95 \%$ and $99.9 \%$ confidence levels of the $\chi^{2}$ statistics. For each source, the red shaded area indicates a region of the parameter space which is not favoured by our comparison with the $24 \mu \mathrm{m}$ flux constraint: spectral solutions falling in this range, all heavily dust extinguished, tend to produce $24 \mu \mathrm{m}$ fluxes in excess of the observed values. Although somewhat model-dependent and unable to associate a formal significance value, our analysis is made relatively robust by our reference to the most extinguished object - Arp 220 - known at the present cosmic time. In particular, we have verified that the adoption of other IR spectral templates, like that of M 82 or other non-standard starburst galaxies, would imply somewhat wider extension of such "disfavoured" regions (in the sense that even lower values of the Av parameter would be inconsistent with the measured $24 \mu \mathrm{m}$ flux or the adopted upper limits). Note that the square shape of the shaded area is shown here only for illustrative purposes.

\subsection{Results of the SED fitting}

In the right panel of Fig. 8 the value of the $\chi^{2}$ is plotted as a function of redshift. Different curves show the result of using different extinction ranges in the SED-fitting procedure with Hyperz $\left(A_{\mathrm{V}}<6\right.$ and $\left.A_{\mathrm{V}}<10\right)$.
As a first result, this analysis indicates the existence of multimodal solutions for the majority of our sample sources (14 out of 20 , \#1, \#2,\#3,\#4,\#5,\#7,\#10,\#12,\#13,\#14,\#15,\#16,\#17,\#19), even accounting for the $24 \mu \mathrm{m}$ constraint: generally these objects have statistically degenerate fits with either a lower-redshift $(z<$ $4)$ and high-extinguished $\left(A_{\mathrm{V}} \sim 3\right) \mathrm{SED}$ or with a higher-redshift $(z>4)$ and lower extinction. Sources \#4 and \#19 have best-fit solutions at $z>\sim 4$.

Six sources (\#6, \#8, \#9, \#11, \#18, and \#20) show a single preferential best-fit solution if we consider the constraint from the MIPS emission and if we limit the analysis to the $95 \%$ confidence level. This number reduces to three sources (\#6, \#8, and \#11) if this constraint is not taken into account. With the exception of the dusty source \#9, all these objects favour a lowextinction solution: four of them lie at $z \sim 4$. Only source \#6 favours a higher redshift best fit $(z \sim 8$, see dedicated discussion in Sect. 6.1). In any case, it should be stressed that, even in the case of single best-fit SEDs, the $z \sim 4$ solutions are only "formally preferred", and alternative lower- $z$ fits cannot be ruled-out with any confidence.

Stellar masses are computed using an adapted version of the Hyperz code that performs SED fitting at a fixed redshift. We give a summary of the best-fit parameters computed by Hyperz in Table 3. Only the solutions matching the $24 \mu \mathrm{m}$ constraint are taken into account in the following. The upper panel of the table reports the objects with a degenerate bimodal redshift solution. In this case, for each parameter we present the lower redshift primary solution (sol. I) and the secondary solution (sol. II) at higher redshift. Moreover, we report a range of values for each physical parameter: the value reported on the left side of the interval corresponds to the $\mathrm{BC} 03$ model expectation, while the value reported on the right side of the interval corresponds to that of the MA05 model. In the lower panel of the table we report the same information for the six sources that we consider to have a single photometric redshift.

For sources with a bimodal behaviour, we compare in Fig. 9 their observed SEDs (open diamonds) with the corresponding low- $z$ best-fit solutions (left panel) reported in Table 3 (sol. I), for the BC03 model. Similarly, in the right panel the observed SEDs are shown together with the corresponding best-fit high$z$ solutions (sol. II in Table 3). Similarly, in Fig. 10 we report the best-fit solution for the six non-degenerate objects (solutions presented in the lower panel of Table 3).

\subsection{Comparison of the Maraston and Bruzual \& Charlot models}

As mentioned in the previous section, we have considered two independent evolutionary population synthesis models, by MA05 and $\mathrm{BC} 03$, in order to check the stability of our results. In Table 3 we report the results for both models. The MA05 model accounts in detail for the contribution of the thermallypulsating asymptotic giant branch (TP-AGB) phase of stellar evolution. The TP-AGB phase is calibrated with local stellar populations and is the dominant source of bolometric and nearIR energy for stellar populations in the age range 0.2 to 2 Gyr. The two models have an underlying different treatment of convective overshooting and red giant branch stars.

In a recent paper, Maraston et al. (2006) test their code on a sample of high-redshift early-type galaxies $(1.4<z<2.5)$, for which the MA05 models indicate younger ages (by a factor up to 6) and lower stellar masses (by $\sim 60 \%$ on average) with respect to results obtained with the models of $\mathrm{BC} 03$. 


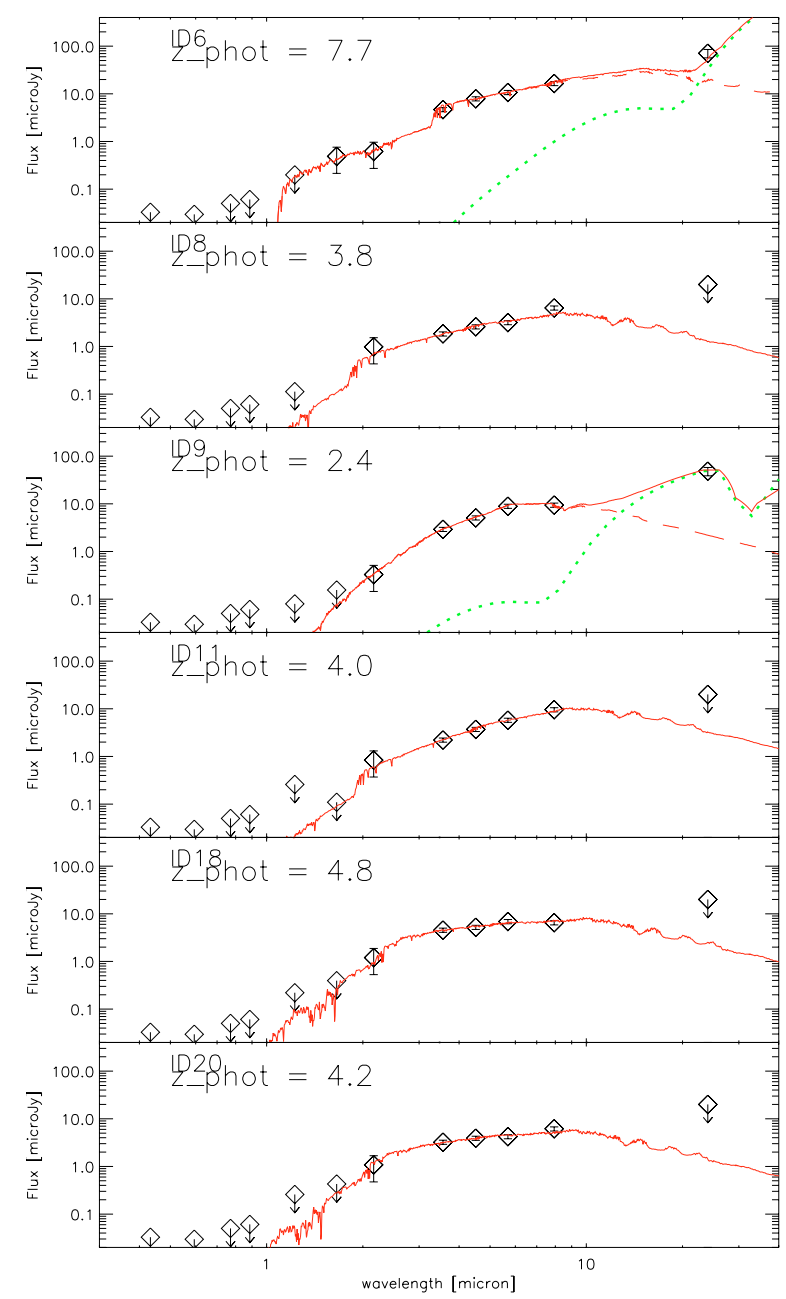

Fig. 10. Best-fit models for the sample source with a single photometric solution. The observed SED of each source (open diamond) is shown with the corresponding best-fit solution (dashed red line) indicated by Fig. 8 for the stellar component (up to $8 \mu \mathrm{m}$ ). The IR part of the spectra has been reproduced with the spectral template of a dusty torus representing the emission of a type-2 AGN (green dotted lines, Fritz et al. 2006 model). The IR spectra have been normalized to match the $24 \mu \mathrm{m}$ measurements. The solid red lines correspond to the sum of the different galaxy components.

For our sample we find that the photometric redshifts obtained with the BC03 models are very similar to those obtained with the MA05 model. The bimodal distributions in the $\left(z, A_{\mathrm{V}}\right)$ parameter space and the $\chi^{2}$ distributions are essentially equivalent for the two independent models. A similar situation applies for the remaining sources. As can be seen in the second and third columns of Table 3 (z-phot: sol. I and sol. II), the scatter in the photometric redshift estimates with MA05 and BC03 is very small at both low and high redshifts. When considering the solution at a lower redshift, the mean ratio of the photometric redshifts derived from $\mathrm{BC} 03$ and MA05, respectively, is 1.04 with an rms of 0.12 , and the mean ratio of the photometric redshifts is 1.07 with an rms of 0.18 for the solution at higher redshift. A slightly larger discrepancy is found for the reddening, with no clear trends.

The most interesting physical parameters are the age and the stellar mass predicted by the two models. In the lower redshift solution the MA05 model predicts older ages than $\mathrm{BC} 03$ for only 2 out of our 14 objects. For the higher- $z$ solutions (including the six non-degenerate objects), 11 out of the 20 sources with a high- $z$ prediction are older for the MA05. As for the stellar masses, we confirm the results of Maraston et al. (2006): in the low- $z$ solutions (mean $z \sim 3.2$ ), the BC03 models predicts $50 \%$ higher masses on average, and a similar trend is found for the high- $z$ (mean $z \sim 5.5)$ fits $(\sim 60 \%)$.

\subsection{Nature of the $24 \mu \mathrm{m}$ emission}

As discussed in Sect. 3.2, the excess $24 \mu \mathrm{m}$ emission often observed in the SEDs of our galaxies (Figs. 9 and 10) requires the presence of either ongoing star formation or AGN activity. In the extinguished low-redshift case $(1<z<3)$, the rest-frame MIPS band samples the emissions by warm dust and PAH molecules. Both a starburst and an AGN emission are then possible explanations of the $24 \mu \mathrm{m}$ flux. We decided in this case to associate the whole mid-IR flux to a starburst process and adopted the Arp 220 SED template (from the spectral library by Polletta et al. 2006). As described in Sect. 3.2, this template was used to compute the IR luminosity $(8-1000 \mu)$ for each object matching the observed $24 \mu \mathrm{m}$ flux (Fig. 9).

At higher redshifts $(z>3.5)$, the low values of the extinction favour a scenario dominated by dust-free sources. In this case the $24 \mu \mathrm{m}$ flux was independently modeled with the emission of a dusty torus reproducing the contribution of a type-2 AGN (Fritz et al. 2006). Again, in Figs. 9 and 10 we show the best-fit solutions at high- $z$, superimposed on the type- 2 spectral templates that reproduce the $S(24 \mu \mathrm{m}) / S(8 \mu \mathrm{m})$ flux ratio better.

\section{X-ray emission and agn activity}

The best independent evidence of nuclear AGN activity in the nuclei of our sources arises if the source is identified as a luminous $\left(L_{X}>10^{42} \mathrm{erg} / \mathrm{s}\right) \mathrm{X}$-ray source. The X-ray data for a total exposure time of $\sim 1$ Ms obtained with the Chandra observatory in the GOODS/CDFS field have been published and made publicly available by Giacconi et al. (2002, see also Alexander et al. 2003). We searched for individual X-ray emission from the 20 objects in the present sample by cross-correlating the IRAC positions of our targets with the positions of X-ray sources as catalogued by Giacconi et al. (2002) and Alexander et al. (2003).

We found that three sources (objects \#6, \#11, and \#13) were individually detected in the X-rays, and the X-ray/IRAC positional difference was smaller than $2^{\prime \prime}$ for all of them. The three sources share similar X-ray properties: all of them have soft Xray spectra and have been detected only in the soft $(0.5-2 \mathrm{keV})$ or full $(0.5-8 \mathrm{keV}) \mathrm{X}$-ray band, with $\sim 13-30$ counts, i.e. at the limiting flux of the X-ray observation $\left(\sim 1 \times 10^{-16} \mathrm{erg} \mathrm{cm}^{-2} \mathrm{~s}^{-1}\right)$. The high values observed for the $\mathrm{X}$-ray to optical flux ratio $\left(F_{\mathrm{X}} / F_{\mathrm{opt}}\right)$ are about 10-100 times higher than generally found for AGNs, which makes these objects "extreme" with respect to the overall X-ray source population. The basic X-ray properties, as drawn from Alexander et al. (2003), are reported in Table 2.

The low number of counts prevented us to perform detailed analyses of the X-ray spectral properties of the sources, especially about the amount of gas absorption, given that at $z \sim 4$ $(\sim 8)$, the $0.5-2 \mathrm{keV}$ observed band corresponds to an intrinsic $2.5-10 \mathrm{keV}$ band (5-20 keV band) and is thus sensitive only to column densities higher than $5 \times 10^{22} \mathrm{~cm}^{-2}\left(5 \times 10^{23} \mathrm{~cm}^{-2}\right)$. With a conservative approach, we derived the intrinsic X-ray luminosities, assuming an unabsorbed power-law with $\Gamma=1.8$ at the best-fit source redshifts, and using XSPEC (Version 11.3.1) to translate the observed count rates into rest frame $0.5-10 \mathrm{keV}$ 
Table 2. X-ray detected sources in our sample.

\begin{tabular}{rlcccccc}
\hline \hline$\#$ & X-ray ID $^{a}$ & $\Delta(X-I R A C)$ & Counts $^{a}$ & Counts $^{a}$ & Counts $^{a}$ & $L_{0.5-10 \mathrm{kev}^{b}}$ & best-fit $z_{\text {phot }}$ \\
\hline $11-4569$ & 217 & $1.90^{\prime \prime}$ & $17.1_{-5.7}^{+6.9}$ & $<15.2$ & $<14.2$ & $1.1_{-0.4}^{+0.4} \times 10^{43}$ & 4 \\
$13-5021$ & $205^{c}$ & $0.21^{\prime \prime}$ & $43.8_{-7.8}^{+9.0}$ & $29.6_{-6.0}^{+7.2}$ & $<15.5$ & $2.7_{-0.5}^{+0.5} \times 10^{43}$ & 4 \\
$6-10945$ & $232^{c}$ & $0.06^{\prime \prime}$ & $17.4_{-6.8}^{+8.0}$ & $13.7_{-5 .}^{+6.2}$ & $<12.8$ & $3.6_{-1.4}^{+1.6} \times 10^{43}$ & $8 \ddagger$ \\
\hline
\end{tabular}

${ }^{a}$ X-ray data from Alexander et al. (2003); ${ }^{b}$ Intrinsic X-ray luminosity at the best-fit photometric redshift, from Table 2; ${ }^{c}$ These sources are also present in the Giacconi et al. (2002) catalogue, as XID \# 217 and XID \#557 with very similar X-ray parameters; ${ }^{\ddagger}$ Following Zheng et al. (2004), Mainieri et al. (2005) associate the observed X-ray emission of \#6 (XID \#557 in their papers) to a faint optical source at a larger distance from the X-ray centroid $\left(\sim 1.4^{\prime \prime}\right)$ and derive a photometric redshift $z=1.81$. However, the detection of a significant $2.2 \mu \mathrm{m}$ signal at the position of the IRAC centroid makes object \#6 the most likely counterpart to the X-ray source (see Fig. 13).

luminosities. The results are reported in Table 2. In all the cases, the X-ray luminosities are in excess of $10^{43} \mathrm{erg} \mathrm{s}^{-1}$, therefore suggesting that nuclear activity is fuelling the central engine.

Seven sources with a solid X-ray detection ( $>25$ counts) and undetected at the limit of the GOODS/CDFS observations have been already reported by Koekemoer et al. (2004, hereinafter K04), who named these objects Extreme X-ray to Optical objects (EXOs). All of them were subsequently detected in the IRAC channels (Koekemoer et al. 2005) with best-fit photometric solutions in the range $2<z<5$ (only one is a candidate $z>7$ AGN). Only one of the three X-ray detected objects in our sample, \#13, is present in Table 1 of K04. The other two escaped the K04 selection because of the low number of X-ray counts $(<20)$. Conversely, of the remaining six objects in the K04 sample, five escaped our selection criteria in the IRAC $3.6 \mu \mathrm{m}$ flux (K04 source \#4, fainter than $1.8 \mu \mathrm{Jy}$ ) or in the $K$-band flux (K04 sources \#2, 3, 5, 7 have $K_{\mathrm{AB}}<=23.5$ in our photometry), while K04 source \#1 was not considered because of a blending problem in the IRAC $3.6 \mu \mathrm{m}$ image. The fainter $K$-band and X-ray fluxes of the X-ray detected objects in our sample with respect to the published EXOs fit well in the narrow $K$-band to X-ray flux correlation shown in Brusa et al. (2005) and can be a result of a higher redshift.

In order to constrain the average X-ray properties of the remaining 17 individually undetected sources, we applied the "stacking technique", following Nandra et al. (2002) and Brusa et al. (2002). For the photometry, a circular aperture with a radius of $2^{\prime \prime}$ centred at the positions of our sources was adopted. The counts were stacked in the standard soft, hard, and full bands $(0.5-2 \mathrm{keV}, 2-7 \mathrm{keV}$, and $0.5-7 \mathrm{keV})$. Extensive Monte Carlo simulations (up to 10000 trials) were carried out by shuffling 17 random positions and using the same photometry aperture ( 2 arcsec). The random positions were chosen to lie in "local background regions" to reproduce the actual background as close as possible. The resulting distributions for the trials are shown in Fig. 11.

No signal was detected in the hard band, for a total effective exposure time of $14.6 \mathrm{Ms}$. An excess of counts above $3 \sigma$ of the expected background level was instead clearly detected in the soft and full bands. Assuming an unobscured $\Gamma=1.8$ power-law spectrum, the stacked count rate in the $0.5-7 \mathrm{keV}$ band $(2.46 \mathrm{e}-$ $6 \mathrm{cts} \mathrm{s}^{-1}$, or $2.3 \times 10^{-17} \mathrm{erg} / \mathrm{cm}^{2} / \mathrm{s}$ ) corresponds to an average $0.5-10 \mathrm{keV}$ rest-frame luminosity of $\sim 1.2 \times 10^{42} \mathrm{erg} \mathrm{s}^{-1}$, at a $z=$ 4 representative redshift. We verified, by splitting the analysis into various subsamples, that this stacked signal was not due to a few objects brighter than the average, but was uniformly spread in the sample.

We plot in Fig. 12 the ratio of the $3.6 \mu \mathrm{m}$ to total X-ray fluxes against the 4.5 to $K$-band monochromatic fluxes. Our sample sources are the $3 \mathrm{X}$-ray detected objects and the 17 individually X-ray undetected sources. For the latter we report

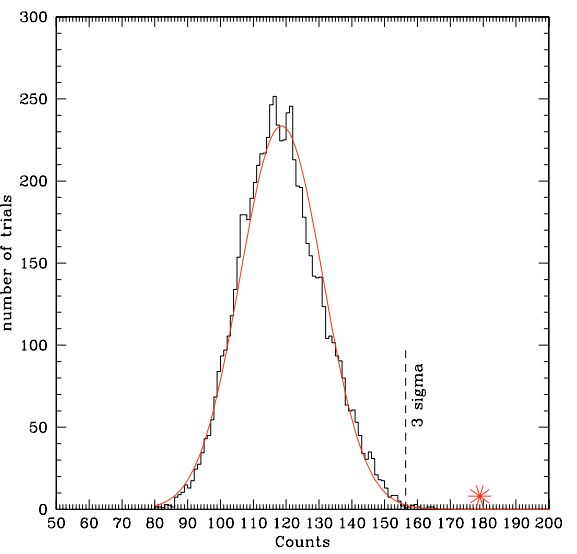

Fig. 11. Distribution of background counts in the full $(0.5-7 \mathrm{keV})$ band. The histogram shows the distribution of background counts in the source cells derived from the Monte Carlo method (10000 trials). The vertical dashed lines show the $3 \sigma$ fluctuation limit: stacked detection with a number of counts higher than these values are considered as "real" detections at the $99.9 \%$ confidence level. The big (red) asterisk represents the counts actually detected from the sample of 17 individually X-ray undetected sources in our sample.

the X-ray to $3.6 \mu \mathrm{m}$ flux ratio assuming for all 17 objects the $2.3 \times 10^{-17} \mathrm{erg} / \mathrm{cm}^{2} / \mathrm{s}$ total average flux detected through the "stacking" analysis. These data are compared with data on an X-ray selected sample by Franceschini et al. (2005), including type- 1 and type- 2 AGNs and type- 2 objects dominated by the host galaxy emission. The lines are the predicted broadband colours as a function of $z$ for a type-1 quasar and a Seyfert-1, a Seyfert-2, and Sb spiral/starburst galaxy.

Figure 12 confirms that 3 of our high $z$ galaxies almost certainly contain an obscured AGN, because they fall in the AGN-dominated region and in view of their large inferred X-ray luminosities. The remaining individually undetected objects fall in a region between that of star-forming galaxies and of AGNs. Figure 12 indicates some probable AGN contributions in the bulk of our high- $z$ galaxy sample. These results are then consistent with those of Figs. 6 and 7.

\section{Candidate massive galaxies at very high redshifts}

\subsection{ID-6: a very massive candidate galaxy at $z \sim 8$ ?}

We mentioned in Sect. 4.1 that the SED of source \#6 formally favours a photometric redshift at $z \sim 8$, with a mass of the order of $M \sim 8-10 \times 10^{11} M_{\odot}$. Given the potential relevance of this finding, we continue analysing this object here. Figure 13 shows the multi-wavelength identification of the source. The Chandra 


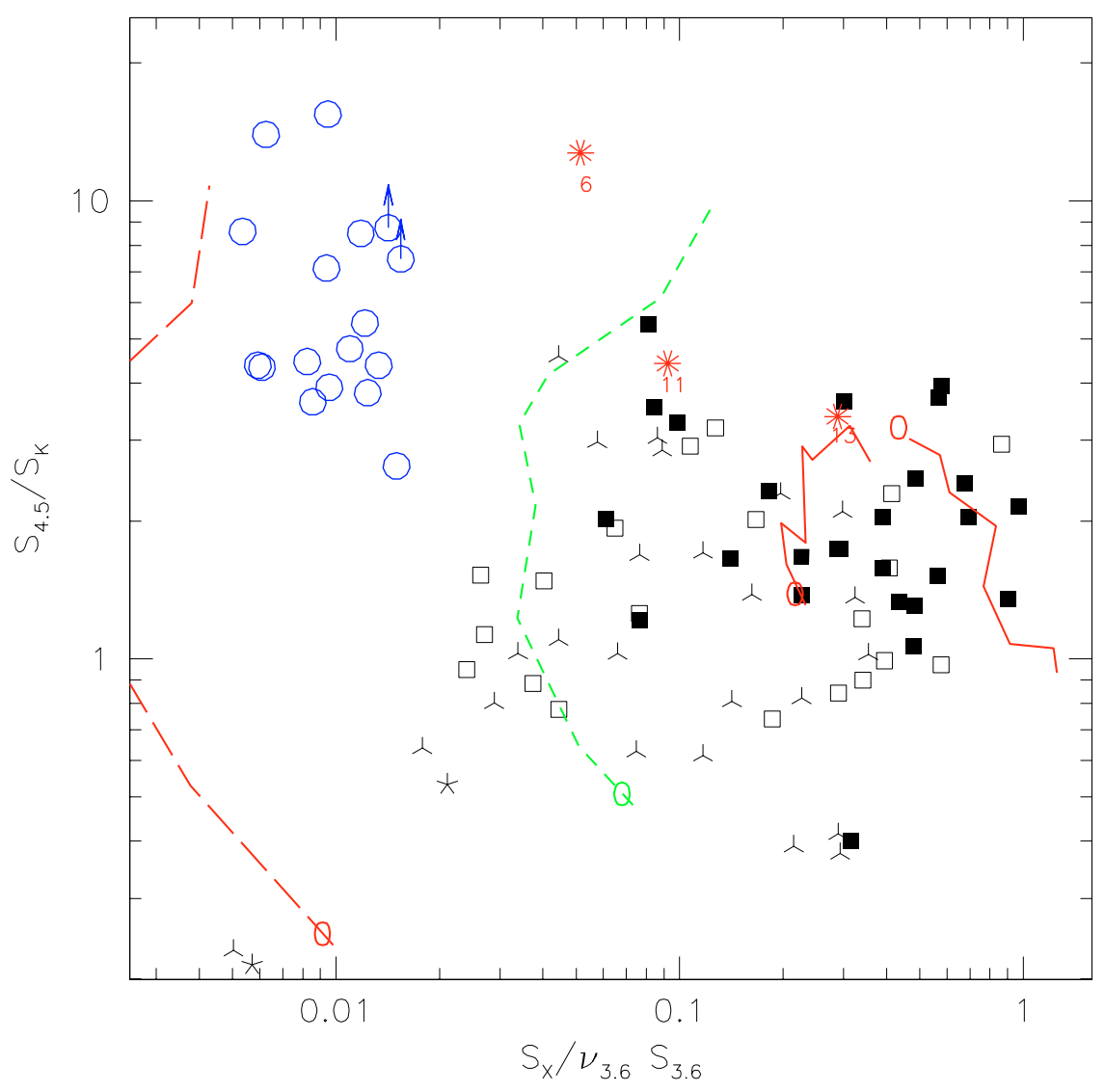

Fig. 12. Plot of the ratio of the $3.6 \mu \mathrm{m}$ to total X-ray fluxes against the 4.5 to $K$-band monochromatic fluxes. Our sample sources are the red asterisks (the $3 \mathrm{X}$-ray detected objects) and the open circles (the 17 individually X-ray undetected sources). For the latter we report the X-ray to $3.6 \mu \mathrm{m}$ flux ratio assuming for all 17 objects the $2.3 \times 10^{-17} \mathrm{erg} / \mathrm{cm}^{2} / \mathrm{s}$ total average flux detected through the "stacking" analysis. These data are compared with data for an X-ray selected sample by Franceschini et al. (2005), including type-1 and type-2 AGNs (filled and open squares) and type- 2 objects dominated by the host galaxy emission (3-legged stars). The lines are the predicted broadband colours as a function of $z$; the colour for $z=0$ corresponds to the point marked with 0 , and lines are drawn from here to points corresponding to $z=0.5,1,1.5,2,2.5,3$, and 3.5. From right to left, the lines correspond to type-1 quasar and Seyfert-1 (the two red solid lines), Seyfert-2 (the green short-dashed line), and Sb spiral/starburst (red long-dashed line).
X-ray object associated to this source, in particular, has been identified differently by various authors. The left panel in the figure shows a $10^{\prime \prime} \times 10^{\prime \prime}$ map of the HST $z$-band overplotted to the IRAC contours. We also report the centroid of the $3.6 \mu \mathrm{m}$ detected source, the position of the X-ray source as reported by Alexander et al. (AID \#232, with the corresponding 2" error box), and all the optical sources detected in the field. The optical counterpart for the X-ray source reported by Zheng et al. (2004) and Mainieri et al. (2005) are also marked. In the right panel of Fig. 13 we report a postage stamp of the $K$-band image, showing that there is a faint but significant $2.2 \mu \mathrm{m}$ source corresponding to the IRAC centroid and excluding the identification with other sources in the optical map (all at $>1.8$ arcsec distances). In addition, the X-ray and IRAC centroids are spatially coincident and clearly indicate the presence of an IR and X-ray source at this position, which is completely absent in the optical images.

We then further investigated the SED properties of this object by combining its IR and X-ray information. The complete observed SED of the source is reported in Fig. 14. In the lower panel we consider the primary solution at $z \sim 7.7$, while in the upper panel we show a secondary solution at $z \sim 3.0$ for comparison. This one was not formally excluded by our analysis, on the basis of the $\chi^{2}$ statistics discussed in Sect. 4.1: a secondary minimum is present at this lower redshift in both panels of Fig. 8 . We also discuss this solution here in this section.

In Fig. 14 we compare the SED data for ID\#6 with various redshifted prototype spectral templates: a type-1 QSO from Elvis et al. (1994), the type-2 QSO NGC6240 (Hasinger et al. 2001), and a typical Seyfert 2 galaxy, all spanning the entire $\mathrm{X}$-ray-to-infrared wavelength range. For both redshift solutions, the models have been normalized to fit the observed $24 \mu \mathrm{m}$ flux. The black continuous-line spectrum from the near-IR to the UV corresponds to the integrated stellar component solution for the galaxy already found in Sect. 4.1 and Figs. 9-10. It is clear that a purely stellar spectrum cannot explain the large observed flux excess at $24 \mu \mathrm{m}$. In the low- $z$ solution, this excess can be explained by dust re-radiation from either a starburst or an AGN, whereas in the high- $z$ hypothesis the only explanation would be a dusty AGN emission. In either case, a type-1 QSO spectrum is entirely inconsistent with the data.

This simple analysis provides interesting hints to the nature of source \#6. In particular, if we refer to the spectral template of NGC 6240 normalized at $24 \mu \mathrm{m}$, the observed X-ray flux is best explained within the higher- $z$ solution, with a redshift of $z \sim 7.7$. The low- $z, z \sim 3.0$, fit instead shows a difficulty in explaining the X-ray data for both the NGC 6240 and the Seyfert-2 templates, since in both cases there is a mismatch of the $24 \mu \mathrm{m}$ to $\mathrm{X}$-ray flux ratio, and excess emission would be expected in the optical/near-IR.

A very interesting comparison of the two redshift solutions is also possible with data on the most luminous Compton-thick quasar recently discovered at $z \sim 2.5$ (SWIRE J104409.95+585224.8) by Polletta et al. (2006). The SED of this object (redshifted and normalized to the observed $24 \mu \mathrm{m}$ flux) is shown in both panels of Fig. 14. Again, for the low- $z$ (lower panel) case, the expected quasar X-ray flux would be too faint and undetectable in X-rays. When redshifted to $z \sim 7.7$, instead, this quasar spectrum turns out to fit the far-IR and X-ray data of source \#6 remarkably well, and the very high redshift brings the unabsorbed hard X-ray spectrum into the observational soft $\mathrm{X}$-ray wavebands. The optical and near-IR emissions would, in any case, be due to the stellar component of the host galaxy (see Sect. 4.1).

The analysis presented in this section provides only qualitative support for the case for a very high-redshift solution for source \#6, which would imply the existence of an extremely 
massive galaxy $\left(M \geq 8 \times 10^{11} M_{\odot}\right)$ at $z \sim 8$. Clearly, given the potentially extraordinary nature of this object, the lower-redshift $z \sim 3$ solution apparent in Fig. 8 must still be considered the more conservative conclusion. Unfortunately, proving this alternative might turn out to be impossible before the advent of JWST and ALMA. The implications of such a finding will be discussed in the next sections.

\subsection{ID-5: The Mobasher et al. candidate high-z galaxy}

Our object \#5 was originally identified and analysed by Mobasher et al. (2005, object HUDF-JD2), and subsequently discussed by Dunlop et al. (2006) and Fontana et al. (2006), among others. The availability of extremely deep photometric data from UDF and near-IR spectroscopy prompted us to perform a more detailed analysis of this source. To this end we adopted a model by Fritz et al. (2007, in preparation; see also Berta et al. 2005; and Poggianti et al. 2000). The model spectrum is obtained by summing SSP spectra that are weighted by different mass values. Each SSP is extinguished with extinction values that are allowed to vary as a function of age (selective dust extinction). Dust is assumed to be distributed in a uniform screen $\left(R_{V}=3.1\right)$. The model also computes the equivalent widths of all relevant interstellar emission lines. Nine SSP spectra have been assumed with ages that were allowed to vary from $10^{6}$ to $\sim 3 \times 10^{9}$, that of the older stars being chosen so as to be consistent with the age of the universe. The best-fit model was found with a $\chi^{2}$ analysis after convolution with the filter response functions. Furthermore, a constraint was used on the $\mathrm{H} \alpha$ emission based on the near-IR spectroscopic observations by Mobasher et al.

Making use of the photometric data by Mobasher et al. we explored both the low- and high- $z$ solutions for source \#5, running our photometric code in the redshift ranges $1<z<3$ and $5<z<8$. In the low- $z$ case, we used the $\mathrm{H} \alpha$ line flux upper limit as a further constraint, which we took to be $9 \times 10^{40} \mathrm{erg} / \mathrm{s}$ from Mobasher et al.

Our code found two best fits with photometric redshifts $z \sim 2.82$ and $z \sim 6.5$ in the two considered intervals. These values are fully consistent with our preliminary investigations (Sect. 4.1). The two solutions are shown in Fig. 15. The upper panel corresponds to the $z=2.82$ solution, the lower panel to that at $z=6.5$. The $\chi^{2}$ statistics sligthly favours the $z \sim 2.82$ solution $\left(\chi^{2}=1.27\right)$, but the $z \sim 6.5$ scenario is equally acceptable $\left(\chi^{2}=1.82\right)$. We find, in particular, that the lack of any $\mathrm{H} \alpha$ emission signal may still be brought into consistency with this low- $z$ interpretation, if selective dust extinction (i.e. extinction as a decreasing function of the age of the various contributing stellar populations) is taken into account.

The $z=6.5$ fit corresponds to a single-burst solution, with an age of $0.3 \mathrm{Gyr}$, that we already found (Sect. 4.1). In Fig. 16 we show the SF history retrieved by the model for the $z=2.82$ solution of Fig. 15. In this case the spectrum is reproduced by SSP ranging from $2 \times 10^{9}$ to $10^{6} \mathrm{yr}$ and extinction as a strong function of the SSP age. The bulk of the stellar mass would be produced between $10^{9}$ and $10^{8} \mathrm{yr}$ before the observation. In view of all these considerations, the lower-redshift solution for galaxy \#5 appears as a more likely explanation.

\section{Main implications}

The results of the analysis presented in this paper are relevant in the general framework of galaxy formation and evolution, and their main implications are outlined here.

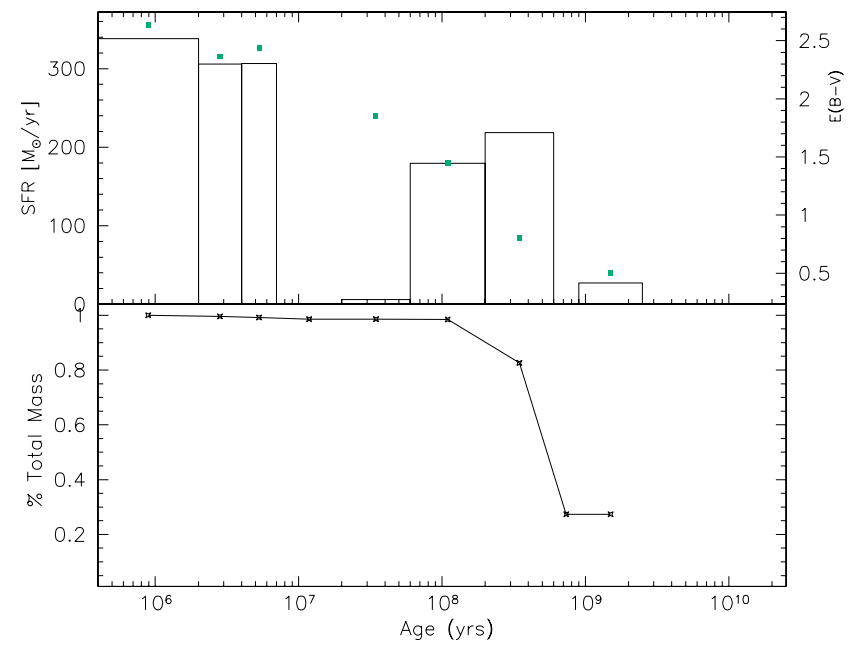

Fig. 16. Upper panel: star formation history of source \#5 for the $z=2.82$ solution. The extinction of each SSP population is also reported (green points) on the right vertical axis. Lower panel: cumulative distribution of the stellar mass assembled in the galaxy as a function of the SSP age.

\subsection{Massive galaxies at $z \sim 4$ ?}

Four sources in our sample (\#8,\#11,\#18, and \#20) show a bestfit photometric redshifts around $z \sim 4$. Solutions at lower redshift produce significantly worse $\chi^{2}$, although they are still formally acceptable. In addition, two more objects (\#4 and \#19) from the bimodal sample present all the acceptable solutions at high redshift, with fits of comparable quality at $z \sim 4$ and $z \sim 6$ and very low extinction. We note here that only source \#4 is detected at $24 \mu \mathrm{m}$.

All these $z \sim 4$ candidates share similar physical properties. Assuming that $z \sim 4$ is the correct interpretation, in most cases the spectral best-fit solutions correspond to either the singleburst model (see Sect. 4.1) or to an exponentially declining SFR with a short decay time-scale $\tau=0.1 \mathrm{Gyr}$. The typical ages are of the order of $\sim 1$ Gyr or younger with the BC03 models ( 1.4 Gyr with the MA05 model). The SEDs are then consistent with galaxies at $z \sim 4$ observed several hundred million years after a powerful burst of star formation producing stellar masses around $10^{11} M_{\odot}$, with both the MA05 and BC03 models, and for a Chabrier IMF.

At lower redshifts, numerous massive $\left(M>10^{11} M_{\odot}\right)$ and old (1-4 Gyr) early-type galaxies have been recently detected from $K$-band selected surveys in the range $1<z<2$ (Cimatti et al. 2004; McCarthy et al. 2004; Glazebrook et al. 2004; Daddi et al. 2005; Saracco et al. 2005; Kriek et al. 2006). Similar detections at slightly higher redshift have also been reported recently ( $z \sim 3.7$, Brammer \& van Dokkum 2007) The photometric modelling generally suggests that these passive systems should have formed all their stars at $z_{f}>3$ in a short burst.

Our sources appear to be among the oldest stellar systems at $z \sim 4$, given that the estimated ages are close to the age of the Universe at that redshift. The post-starburst nature of our $z \sim 4$ sources and their typically high stellar masses around $M \sim 10^{11} M_{\odot}$ suggest that they could be seen as the progenitors of the most massive spheroids that are observed around $z \sim 2$. Moreover, as already mentioned, the $z \sim 4$ population is mostly undetected at $24 \mu \mathrm{m}$, making the passively evolving nature of these sources a plausible hypothesis. 
One of these galaxies (\#11, see Sect. 5 and Fig. 12) has been detected in X-rays and is consistent with hosting an obscured AGN. The X-ray emissions from all the other 5 objects mentioned in this section were investigated by us via a stacking analysis, which has detected significant average signal (at better than $3 \sigma)$.

\subsection{Extremely dusty starbursts at $2<z<3$ ?}

As mentioned in Sect. 4.1, the remaining fraction of the sources in our sample show strong bimodality in the photometric redshift solutions. We also showed there that these objects are consistent with a population of heavily dust-enshrouded starbursts at redshift $2<z<3$, since only a few of them are undetected at $24 \mu \mathrm{m}$. Based on this flux, we have computed the instrinsic bolometric luminosity, $L_{\mathrm{IR}}=L(8-1000 \mu \mathrm{m})$ (see discussion in Sect. 4.1).

The derived luminosities qualify most of these sources as ULIRGs $\left(L_{\mathrm{IR}}>10^{12} L_{\odot}\right)$. Assuming that the mid-IR emission is mostly contributed by star-formation processes, we can translate the IR luminosity into a SFR by adopting the standard relation of Kennicutt et al. (1998). The derived SFRs have a median value $\sim 3000 M_{\odot} / \mathrm{yr}$, even greater than the typical value of submillimetre selected galaxies at $z \sim 2.2\left(\sim 1700 M_{\odot} / \mathrm{yr}\right.$, Chapman et al. 2004). The high bolometric luminosities $\left(L_{\mathrm{IR}}>10^{12} L_{\odot}\right)$ implied by our photometric analysis and the instrinsic large extinction required $\left(A_{\mathrm{V}} \sim 2-4\right)$ make our sample very similar to the optically obscured sources identified by Houck et al. (2005) and could represent the most extreme cases of dusty galaxies detected until now.

It seems likely, however, that part of this IR emission may be due to an AGN contribution, if we consider that the average $\mathrm{X}$-ray signal and the corresponding X-ray-to-3.6 $\mu \mathrm{m}$ flux ratios in Fig. 12 reveal X-ray activity in excess of what is expected from star-forming galaxies.

In spite of the intrinsic uncertainties in the photometric solutions, we computed the contribution of these sources to the SFR density of the Universe by assuming that their far-IR emission is mainly contributed by star formation processes. We used the $1 / V_{\max }$ estimator (see more details in Sect. 8). Even including all objects with a photometric- $z$ solution in the redshift range $1<z<3.5$ and detected at $24 \mu \mathrm{m}(\# 1, \# 2, \# 3$, \#5, \#7, \#9, $\# 10$, \#14, and \#16), we found that these sources missed by optical surveys contributes only $\sim 20 \%$ to the global SFR density at $1<z<3.5$. We obtained a value of $\rho_{\mathrm{SFR}}=0.026 \mathrm{M}_{\odot} / \mathrm{yr} / \mathrm{Mpc}^{3}$, see Perez-Gonzalez et al. (2005) for a recent compilation of the Lilly-Madau diagram of IR-selected galaxies.

\subsection{Massive evolved post-starburst galaxies at $z>6$ ?}

The fourteen sources with bimodal photometric redshift solutions (upper panel in Table 3 ) all have a secondary best fit in the redshift range $4.5<z<9$, see Sect. 4.1 and Fig. 10, and appear to have been detected at less than $1 \mathrm{Gyr}$ after a powerful burst of star formation producing a stellar mass of the order of $\sim 10^{11} M_{\odot}$.

Two sources in particular (\#4 and \#19), have formally better solutions at $z>6$ and predicted masses $M>10^{11} M_{\odot}$ from both evolutionary synthesis models. In addition, source \#6 has a best fit at $z \sim 7.7$. These three sources have predicted ages for their stellar populations of $<500 \mathrm{Myr}$ at their estimated photometric redshifts of $6.8,6.2$, and 7.7, in that order. If all these sources were at $z>6$ galaxies, then these would have formed the bulk of their massive stellar populations at very high redshift, $z>9$, in a period of several tens of million years before entering a quiescent phase. This would have required an enormous star-formation activity, with rates of the order of 1000-6000 $M_{\odot} / \mathrm{yr}$.

Two objects (\#4 and \#6) have MIPS detections at $24 \mu \mathrm{m}$, and one is bright in X-rays (\#6), which could only be interpreted at such high redshifs as the signature of a luminous type- 2 obscured quasar within their nucleus; this conclusion was proposed also by Mobasher et al. for source \#5/HUDF-JD2. Mobasher et al. also estimated the size of the dark-matter halo required to host the stellar mass of source \#5/HUDF-JD2 and found a value of $4 \times 10^{12} M_{\odot}$ (if the estimated stellar mass of the source is $6 \times$ $10^{11} M_{\odot}$ at $z \sim 6.5$ and a Salpeter IMF is considered).

Assuming such high redshifts and the associated huge energetics from both star formation and black-hole gravitational accretion, this would have played an important role in the reionization of large surrounding volumes of the Universe, starting the process at redshifts as high as $z \sim 15$ (Panagia et al. 2005). The detection of three comparably massive sources within the same small sky region $(\sim 130$ square arcmin) would support the hypothesis that the reionization of the Universe might be dominated by such massive galaxies.

However, two of the very high-redshift galaxy candidates have lower- $z$ best-fit solutions of comparable quality, which are obviously much more likely. Only for source \#6 the high- $z$ $(z \sim 8)$ fit is formally preferred to the low $-z$ one (Sect. 6.1). At this stage, and waiting for more decisive future observations with JWST and ALMA, we can only set a (stringent) limit to the existence of massive evolved galaxies at very high redshifts.

\section{Contribution to the comoving stellar mass density at high $z$}

Given the significance of the massive galaxy population at $z \sim 4$ (Sect. 7.1), and even that of the $z \sim 6$ candidates (Sect. 7.3), it is worthwhile to attempt to compare their contribution to the global comoving stellar mass density with those already derived from other independent surveys at those redshifts. We computed it by using the $1 / V_{\max }$ estimator (see e.g. Franceschini et al. 2006, for an application). Our evaluation should be considered a lower limit to the comoving mass density because our sample is not purely flux-limited (we excluded a priori blended sources and applied various colour limits, see Sect. 2.4).

(A) $z \sim 4$. We limited our analysis to the more massive galaxies, those with $M>10^{11} M_{\odot}$. For the galaxies described in Sect. 7.1, we computed for each sources the effective co-moving volume of the survey (defined by the survey area and the redshift interval, $z_{\min }<z<z_{\max }$, within which each of the $z \sim 4$ candidates could have been detected, with $z_{\min }=3.7$ ). To determine $z_{\max }$, we took the best-fit SED to each candidate and redshifted it until its flux fall below our limit $\left(S_{3.6}=1.8 \mu \mathrm{Jy}\right)$ and took as $z_{\max }$ the minimum of the corresponding redshift and our upper boundary of the redshift interval, $z=4.7$ (for most of our objects $z_{\max }=4.7$, corresponding to a survey volume $V_{\max }=3.9 \times 10^{5} \mathrm{Mpc}^{3}$ for our adopted cosmology). We derived two estimates of the mass density based on the MA05 and BC03 spectral solutions reported in Table 3 .

We report in Fig. 17 the contribution of our galaxies to the stellar mass function at $z \sim 4$. The shaded region corresponds to the data reported by Drory et al. (2005), of which we considered an average of their mass functions in the two redshift bins $3<z<4$ and $4<z<5$. The lower and upper envelopes of the region correspond to the mass functions derived by Drory et al. in the GOODS/CDFS and FORS deep field, respectively. We report our estimated contribution to the mass function by our 


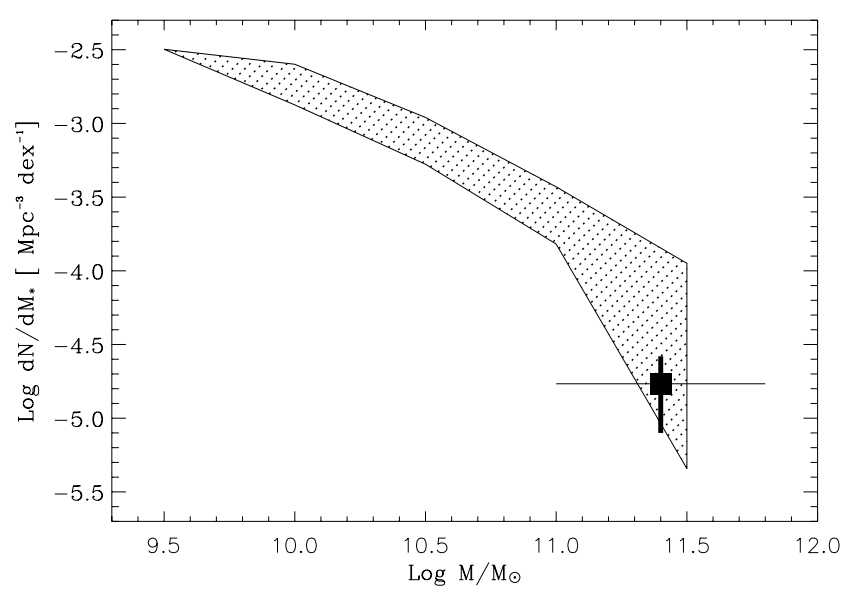

Fig. 17. The stellar mass function at $z \sim 4$. The dashed region corresponds to the data reported by Drory et al. (2005). We have considered the mean value of the mass function in the two redshift bins $3<z<4$ and $4<z<5$. The lower and upper envelopes of the dashed region correspond to the values derived in the GOODS/CDFS and Fors Deep Field, respectively, by Drory et al. (2005). The filled square marks our estimated lower limit to the mass function at $z \sim 4$ for our sample: the range spanned by the vertical arrow corresponds to the range of values computed with MA05 (lower boundary) and BC03 (upper boundary) models.

$z=4$ galaxies. The vertical errorbar corresponds to our overall uncertainty, in which the lower limit corresponds to stellar mass values computed with MA05 (based only on 3 objects with favoured photo- $z$ at $z=4, \# 11$, \#18, and \#20), while the upper bound is based on BC03 fits (including 3 sources with favoured photo- $z$ at $z=4, \# 11, \# 18$, and \#20, and anadditional 7 galaxies with bimodal photo- $z$ solutions, one of which solutions is within $3.7<z<4.7$, \#1,\#3,\#4, \#12,\#14,\#15, and \#19, see Sect. 7.2 above).

Figure 18 compares our estimated number density at $z=4$ of galaxies that are more massive than $M=10^{11} M_{\odot}$ with literature data at different redshifts. We also report at $z=5.3$ the estimate for Lyman Break galaxies (LBGs) with $M>10^{11} M_{\odot}$ recently derived by McLure et al. (2006) and the GOODS/CDFS $K$-band selected sample of Caputi et al. (2006) and of Drory et al. (2005) in the same mass range. Altogether, our very red galaxies account for a large fraction of the galaxy mass density at $z=4$ and are among the most massive galaxies currently known at such redshifts.

(B) $z \sim 6$. As already discussed, our conclusions at higher redshift are subject to major uncertainties because of the degeneracy in the solutions for half of our sample (Sects. 7.3 and 4.1). However, a maximal and minimal case for the contribution to the stellar mass density implied by our analysis may be interesting. For the galaxies described in Sect. 7.3 with a photometric solution in the redshift range $5.5<z<8.5$, we then estimated such contribution as for the $z \sim 4$ case; again, we limited our analysis to the more massive galaxies, those with $M>10^{11} M_{\odot}$. The result of this computation is reported in Fig. 18 at $z \sim 6.7$. The vertical thick errorbar corresponds to the overall uncertainty, in which the lower limit corresponds to stellar mass values computed only with source \#6 (the unique object in our sample with a clearly favoured solution at high redshift), while the upper bound includes six sources (\#4, \#5, \#6, \#10, \#12, \#16, and \#19).

The search for $z \sim 4$ galaxies has so far been mainly performed with the traditional Lyman dropout colour selection technique (Steidel et al. 1999). LBGs are associated with starburst

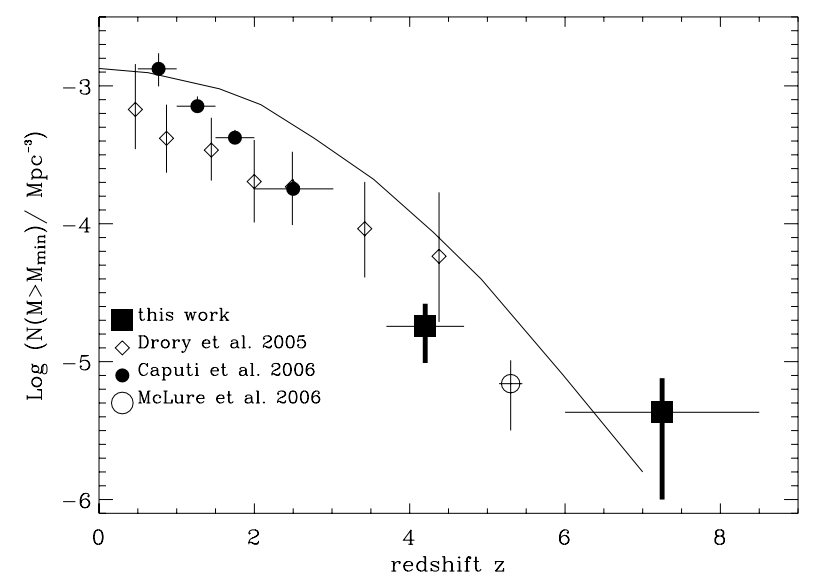

Fig. 18. Redshift evolution of the number density of galaxies with stellar masses $M>10^{11} M_{\odot}$ : our data are reported with the same symbol as in Fig. 17, at $z=4$. The open circle at $z=5.3$ is the estimate for LBGs with $M>10^{11} M_{\odot}$ recently derived by McLure et al. (2006). The filled circles are the estimated number densities of galaxies with $M>10^{11} M_{\odot}$ from the GOODS/CDFS $K$-band selected sample of Caputi et al. (2006). We also report the compilation by Drory et al. (2005, open diamonds) in the same mass range. The asteriks marks the number density of red and dead galaxies with $M>0.5 \times 10^{11} M_{\odot}$ at $2<z<3$ detected by Labbè et al. (2005). The solid line shows the redshift evolution of the number density of dark matter halos with masses of $M>2 \times 10^{12} M_{\odot}$, matching the number density of galaxies with $M>10^{11} M_{\odot}$ at $z=0$ (adapted from Fig. 5 of McLure et al. 2006).

galaxies at high redshifts, identified by the colours of their far ultraviolet spectral energy distribution around the 912 A Lyman continuum discontinuity (Giavalisco et al. 2002). It has been recently suggested that $60 \%$ of the stellar mass at $z \sim 5$ is missed by the traditional drop-out selection technique (Stark et al. 2006), which excludes high-redshift galaxies that are too red in the rest-frame UV to fall under the LBG selection (McLure et al. 2006).

A fraction of our galaxies might represent a complementary sample of mature and red galaxies that, by definition, would not be selected by the Ly break or other methods making use of the optical-UV rest-frame emission. Our results in Figs. 17 and 18 show that the contributions by this new, previously unaccounted, population is definitely non-negligible if not to dominate the galaxy mass function at the highest $z$. These objects, all escaping detection by published optically-selected or $K$-band selected samples, would almost double the estimated value for the highend of the galaxy mass function (Drory et al. 2005; Fontana et al. 2006) at that redshift.

Would this reassessment of the galaxy mass function still be consistent with standard $\Lambda$ CDM model expectations at such high redshifts? Following McLure et al. (2006) and Dunlop et al. (2006), we report in Fig. 18 the redshift evolution of the number density of dark-matter halos with masses of $M>2 \times 10^{12} M_{\odot} \mathrm{TT}$, matching the number density of galaxies with $M>10^{11} M_{\odot}$ at $z=0$ (adapted from Fig. 5 of McLure et al. 2006). The proposed model corresponds to a ratio between halo and stellar mass of 20 (see discussion in McLure et al. 2006). Looking at Fig. 18 we can see that the number density of our massive sources at $z \sim 4$ is a factor $\sim 2-8$ (with MA05 and BC03, respectively) lower than the predicted number of dark-matter halos. Even if our data is only a lower limit, this implies that our estimated number density of massive galaxies at $z \sim 4$ is still consistent with the current predictions of $\Lambda \mathrm{CDM}$ models for the hierarchical formation of cosmic structures. 


\section{Discussion and conclusions}

Recent observational progress has revealed the existence of massive structures (galaxies and galaxy aggregates) at high redshift much more frequently than originally supposed. Indeed, the problem with all attempts to predict the origin of galaxies from first principles is dealing with the very complex physical processes involving baryons (e.g. Somerville et al. 2001, 2004). Observations have also shown an unexpected trend for the most massive part of the galaxy mass function to be put in place first and for lower mass galaxies to keep forming stars at lower redshifts (Cowie et al. 1996; Franceschini et al. 1998, 2006; Bundy et al. 2005).

At the present stage, however, the timing for and the physical processes accompanying the emergence of massive galaxies with cosmic time, which would be so informative for our understanding of galaxy formation, are essentially unknown. A problem here is that the most efficient tool for identifing very high-redshift galaxies, the Ly-dropout technique, is not sensitive to galaxy mass but rather to UV flux.

The advent of sensitive imagers in the near-IR atmospheric JHK bands and particularly of the Spitzer IRAC space facility have started to provide new powerful selection tools that are more sensitive to the host stellar mass. Several reports have recently appeared about searches for massive galaxies at $z>4$ and the evolution of the stellar mass function (Stark \& Ellis 2006; Yan et al. 2006; McLure et al. 2006; Grazian et al. 2006; Fontana et al. 2006). Most of these have in any case exploited the Ly-dropout approach and pushed it to the reddest optical bands for the highest $z$ characterisation and used IRAC data to constrain the stellar mass.

Dunlop et al. (2006; see also Mobasher et al. 2005) followed a complementary method of carrying out an extensive SED-fitting analysis of large flux-limited samples without preconceived assumptions about the rest-frame spectrum of the candidates and by using as reference a $K$-band selection with $K_{\mathrm{S}}<23.5$. They conclude in favour of a lack of evidence for very massive galaxies to be in place at $z>4$.

We follow a similar approach here, but extend it to a galaxy selection based on the most sensitive Spitzer $3.6 \mu \mathrm{m}$ IRAC images in the $130 \mathrm{arcmin}^{2}$ GOODS CDFS, a $K$-selection complementing that of Dunlop et al. ( $K>23.5 \mathrm{AB} \mathrm{mag})$, and nondetection in any GOODS optical bands. We were aiming by these means at detecting the most massive and highest redshift galaxies in the field to the $3.6 \mu \mathrm{m}$ limiting flux and at keeping equally sensitive to dusty star-forming and massive evolved galaxies at high $z$.

Our results in terms of the stellar mass and redshift for such extreme $3.6 \mu \mathrm{m}$ selected galaxies are summarised in Fig. 19, where we plot the stellar mass versus photometric reshift estimated from SED fitting. We show with different symbols data for the 6 galaxies with favoured single photo- $z$ solution in our discussion and for the remaining 9 galaxies with both the lower$z$ solutions and the higher- $z$ ones.

Given the uncertainties in the photometric redshift solutions, our results are consistent with previous reports, and point towards very few of the galaxies in the field being found at $z>6$, with only one such extreme case (galaxy \#6) being formally indicated (though a lower- $z$ fit is still acceptable). Two other, similarly massive, galaxies are consistent with $z>6$ solution but do not require it. Remarkably, and similar to what was found by Dunlop et al., two of the three candidates have solid detections in the Spitzer MIPS $24 \mu \mathrm{m}$ band. However, differently from Dunlop et al., we do not consider this as necessarily an argument

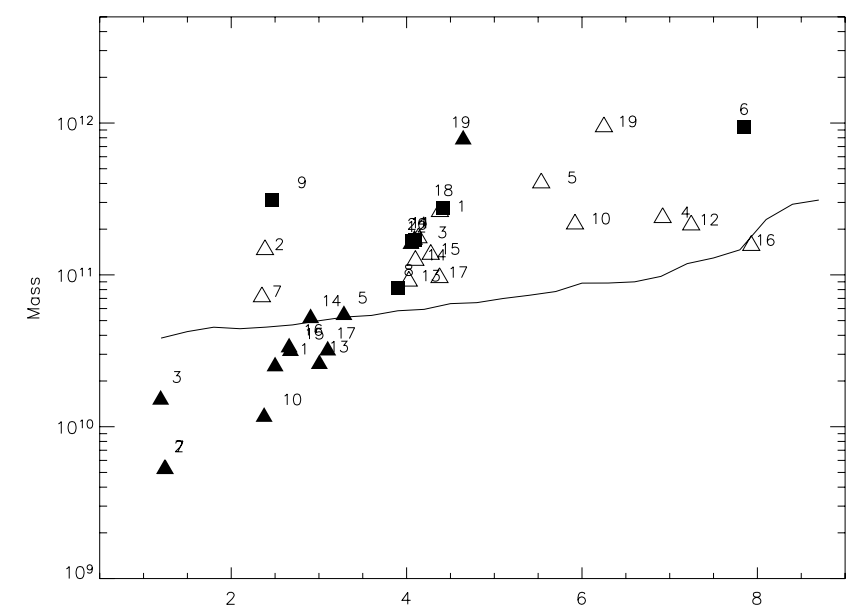

Fig. 19. Plot of the stellar mass versus photometric redshift, summarising the results of our selection for very high- $z$ galaxies based on selection at IRAC $3.6 \mu \mathrm{m}$ in the $130 \mathrm{arcmin}^{2}$ GOODS CDFS field, a $K$-band flux fainter than $K=23.5 \mathrm{AB}$ mag, and non-detection in any GOODS optical bands. For 11 galaxies shown in filled squares we show the unique photo- $z$ solution. For the remaining 9 galaxies we both report as filled triangle the lower- $z$ fit and with the open triangle the higher- $z$ one. The continuous line corresponds to the mass of a 1 Gyr-old SSP detectable at the survey limit. The selection procedure clearly tends to detect massive evolved galaxies around $z \sim 4$.

in favour of a lower- $z$ case, because the presence of an obscured AGN could easily explain it. Indeed, we have found some evidence of (optically) hidden AGNs in the majority of our sample of very red high-redshift galaxies from the ultra-deep Chandra X-ray data (Fig. 12), which adds to the frequently observed midIR $24 \mu \mathrm{m}$ excess. We concluded from this that there might be room for a substantial contribution to re-ionization to occur in relation with star-formation and AGN activity in massive galaxies.

One major result of our analysis is the potential existence of a candidate population of massive galaxies detected around redshift 4 . The majority of our sample galaxies (14 out of 20) have a photo- $z$ solution at $3.7<z<4.7$, and 4 of them have best-fit solutions in this redshift interval (not formally unique, however). Hence, in spite of the small numbers, a galaxy population undetected in the optical and extremely faint in the $K$-band appears to possibly dominate the massive end of the galaxy mass function at $z=4$. These objects, all escaping detection by published optically-selected or $K$-band selected samples, would almost double the estimated value for the high end of the galaxy mass function (Drory et al. 2005; Fontana et al. 2006) at that redshift.

Several of these evolved $z \sim 4$ galaxies (none of the 4 with a robust $z=4$ solution, but 4 of the 7 with "secondary" solution at this $z$ ) display strong excess emission at $24 \mu \mathrm{m}$. This result is similar to what is reported by Dunlop et al. (2006) for their selected, very high- $z$ population. Due to the high redshifts, this would correspond to rest-frame emission at $4-5 \mu \mathrm{m}$, so would be difficult to explain purely as dust reprocessing by star-forming regions. Again this result requires rather common AGN activity in these high- $z$ evolved galaxies. A support for this interpretation comes from the deep X-ray data, revealing 2 galaxies with bonafide $z=4$ photometric- $z$ to have clear AGN-like emissions (with $L_{\mathrm{X}}>10^{43} \mathrm{erg} / \mathrm{s}$ ) and the remaining objects also showing excess X-ray flux (Fig. 12), although at a lower level. Some evidence of probable AGN contributions at 8 and $24 \mu \mathrm{m}$ was also found directly in the colour-colour plots of Figs. 6 and 7. 
This widespread association of very high- $z$ galaxies with trace-obscured AGN activity might confirm the emergent view (e.g. De Lucia et al. 2005; Bower et al. 2006; Granato et al. 2004) that AGN feedback could have systematically influenced the shaping of the galaxy mass function during the epoch of galaxy formation.

Acknowledgements. This work is based on observations made with the Spitzer Space Telescope, which is operated by the Jet Propulsion Laboratory, California Institute of Technology under NASA contract 1407. ESO/GOODS observations were carried out using the Very Large Telescope at the ESO Paranal Observatory under Program ID(s): LP168.A-0485. The NASA/ESA Hubble Space Telescope is operated by the Association of Universities for Research in Astronomy (AURA), Inc., under NASA contract NAS5-26555. We thank the referee for his/her detailed comments and suggestions that improved the quality of our work. We warmly thank Claudia Maraston from providing us with her evolutionary model predictions in electronic form and Alvio Renzini, Stefano Berta, and Vincenzo Mainieri for useful discussions.

\section{References}

Abraham, R. G., Glazebrook, K., McCarthy, P. J., et al. 2004, AJ, 127, 2455 Alexander, D. M., Bauer, F. E., Brandt, W. N., et al. 2003, AJ, 126, 539 Allard, F., Hauschildt, P. H., Alexander, D. R., Tamanai, A., \& Schweitzer, A. 2001, ApJ, 556, 357

Berta, S., Fritz, J., Franceschini, A., Bressan, A., \& Pernechele, C. 2003, A\&A, 403, 119

Bertin, E., \& Arnouts, S. 1996, A\&AS, 117, 393

Bertoldi, F., Cox, P., Neri, R., et al. 2003, A\&A, 409, L47

Bolzonella, M., Miralles, J.-M., \& Pelló, R. 2000, A\&A, 363, 476

Bouwens, R., \& Illingworth, G. 2006, New Astron. Rev., 50, 152

Bower, R. G., Benson, A. J., Malbon, R., et al. 2006, MNRAS, 370, 645

Brammer, G. B., \& van Dokkum, P. G. 2007, ApJ, 654, L107

Brusa, M., Comastri, A., Daddi, E., et al. 2002, ApJ, 581, L89

Brusa, M., Comastri, A., Daddi, E., et al. 2005, A\&A, 432, 69

Bruzual, G., \& Charlot, S. 2003, MNRAS, 344, 1000

Bundy, K., Ellis, R. S., \& Conselice, C. J. 2005, ApJ, 625, 621

Calzetti, D., Armus, L., Bohlin, R. C., et al. 2000, ApJ, 533, 682

Caputi, K. I., McLure, R. J., Dunlop, J. S., Cirasuolo, M., \& Schael, A. M. 2006, MNRAS, 366, 609

Chapman, S. C., Smail, I., Windhorst, R., Muxlow, T., \& Ivison, R. J. 2004, ApJ, 611,732

Cimatti, A., Pozzetti, L., Mignoli, M., et al. 2002, A\&A, 391, 1

Cimatti, A., Daddi, E., Cassata, P., et al. 2003, A\&A, 412, L1

Cimatti, A., Daddi, E., Renzini, A., et al. 2004, Nature, 430, 184

Cioni, M.-R. L., Girardi, L., Marigo, P., \& Habing, H. J. 2006, A\&A, 452, 195

Cowie, L. L., Songaila, A., Hu, E. M., \& Cohen, J. G. 1996, AJ, 112, 839

Daddi, E., Cimatti, A., Renzini, A., et al. 2004, ApJ, 600, L127

Daddi, E., Renzini, A., Pirzkal, N., et al. 2005, ApJ, 626, 680

Dannerbauer, H., Lehnert, M. D., Lutz, D., et al. 2004, ApJ, 606, 664

De Lucia, G., Springel, V., White, S. D. M., Croton, D., \& Kauffmann, G. 2006, MNRAS, 366, 499

Dietrich, M., Hamann, F., Shields, J. C., et al. 2003, ApJ, 589, 722

Drory, N., Salvato, M., Gabasch, A., et al. 2005, ApJ, 619, L131

Dunlop, J. S., Cirasuolo, M., \& McLure, R. J. 2006, [arXiv:astro-ph/0606192]

Elvis, M., Wilkes, B. J., McDowell, J. C., et al. 1994, ApJS, 95, 1

Fan, X., Strauss, M. A., Schneider, D. P., et al. 2003, AJ, 125, 1649

Fan, X., Hennawi, J. F., Richards, G. T., et al. 2004, AJ, 128, 515

Fontana, A., Pozzetti, L., Donnarumma, I., et al. 2004, A\&A, 424, 23

Fontana, A., Salimbeni, S., Grazian, A., et al. 2006, [arXiv: astro-ph/0609068]

Fan, X., Wilkes, B. J., \& McDowell, J. C. 2006, Mem. Soc. Astron. It., 77, 635

Franceschini, A., Silva, L., Fasano, G., et al. 1998, ApJ, 506, 600

Franceschini, A., Manners, J., Polletta, M. C., et al. 2005, AJ, 129, 2074

Franceschini, A., Rodighiero, G., Cassata, P., et al. 2006, A\&A, 453, 397

Franx, M., Labbé, I., Rudnick, G., et al. 2003, ApJ, 587, L79

Frayer, D. T., Chapman, S. C., Yan, L., et al. 2004, ApJS, 154, 137

Freudling, W., Corbin, M. R., \& Korista, K. T. 2003, ApJ, 587, L67

Fritz, J., Franceschini, A., \& Hatziminaoglou, E. 2006, MNRAS, 366, 767
Giacconi, R., Zirm, A., Wang, J., et al. 2002, ApJS, 139, 369

Giavalisco, M. 2002, ARA\&A, 40, 579

Giavalisco, M., Ferguson, H. C., Koekemoer, A. M., et al. 2004, ApJ, 600, L93

Glazebrook, K., Abraham, R. G., McCarthy, P. J., et al. 2004, Nature, 430, 181

Granato, G. L., De Zotti, G., Silva, L., Bressan, A., \& Danese, L. 2004, ApJ, 600,580

Grazian, A., Fontana, A., de Santis, C., et al. 2006, A\&A, 449, 951

Jarrett, T. H., Dickman, R. L., \& Herbst, W. 1994, ApJ, 424, 852

Hasinger, G., Altieri, B., Arnaud, M., et al. 2001, A\&A, 365, L45

Hopkins, A. M., \& Beacom, J. F. 2006, [arXiv:astro-ph/0601463]

Houck, J. R., Soifer, B. T., Weedman, D., et al. 2005, ApJ, 622, L105

Kennicutt, R. C., Jr. 1998, ARA\&A, 36, 189

Knudsen, K. K., van der Werf, P., Franx, M., et al. 2005, ApJ, 632, L9

Koekemoer, A., Alexander, D. M., Bauer, F. E., et al. 2004, ApJ, 600, L123

Koekemoer, et al. 2005, Growing black holes: accretion in a cosmological context. Proceedings of the MPA/ESO/MPE/USM Joint Astronomy Conference held at Garching, Germany, 21-25 June 2004, ed. A. Merloni, S. Nayakshin, \& R. A. Sunyaev, ESO Astrophys. Symp., 120

Kriek, M., et al. 2006, ApJ, 649, L71

Im, M., Yamada, T., Tanaka, I., \& Kajisawa, M. 2002, ApJ, 578, L19

Labbé, I., Huang, J., Franx, M., et al. 2005, ApJ, 624, L81

Lacy, M., Storrie-Lombardi, L. J., Sajina, A., et al. 2004, ApJS, 154, 166

Lacy, M., Wilson, G., Masci, F., et al. 2005, ApJS, 161, 41

Le Fèvre, O., Paltani, S., Arnouts, S., et al. 2005, Nature, 437, 519

Madau, P. 1995, ApJ, 441, 18

Mainieri, V., Rosati, P., Tozzi, P., et al. 2005, A\&A, 437, 805

Mannucci, F., Buttery, H., Maiolino, R., Marconi, A., \& Pozzetti, L. 2006, [arXiv: astro-ph/0607143]

Maraston, C. 2005, MNRAS, 362, 799

Maraston, C., Daddi, E., Renzini, A., Cimatti, A., Dickinson, M., et al. [arXiv:astro-ph/0604530]

Mayya, Y. D., Bressan, A., Rodríguez, M., Valdes, J. R., \& Chavez, M. 2004, ApJ, 600, 188

McCarthy, P. J., Le Borgne, D., Crampton, D., et al. 2004, ApJ, 614, L9

Maiolino, R., Nagao, T., Marconi, A., et al. 2006, [arXiv: astro-ph/0603261]

McLure, R. J., Cirasuolo, M., Dunlop, J. S., et al. 2006, [arXiv: astro-ph/0606116]

Mobasher, B., Dickinson, M., Ferguson, H. C., et al. 2005, ApJ, 635, 832

Moustakas, L. A., Casertano, S., Conselice, C. J., et al. 2004, ApJ, 600, L131

Nandra, K., Mushotzky, R., Arnaud, K., et al. 2002, ApJ, 576, 625

Omont, A., McMahon, R. G., Cox, P., et al. 1996, A\&A, 315, 1

Omont, A., Beelen, A., Bertoldi, F., et al. 2003, A\&A, 398, 857

Panagia, N., Fall, S. M., Mobasher, B., et al. 2005, ApJ, 633, L1

Papovich, C., Moustakas, L. A., Dickinson, M., et al. 2006, ApJ, 640, 92

Peng, C. Y., Impey, C. D., Ho, L. C., Barton, E. J., \& Rix, H.-W. 2006, ApJ, 640, 114

Pérez-González, P. G., Pablo, G., Rieke, G. H., et al. 2005, ApJ, 630, 82

Poggianti, B. M., \& Wu, H. 2000, ApJ, 529, 157

Poggianti, B. M., Bressan, A., \& Franceschini, A. 2001, ApJ, 550, 195

Polletta, M. D. C., Wilkes, B. J., Siana, B., et al. 2006, ApJ, 642, 673

Richards, et al. 2006 [arXiv: astro-ph/060155]

Robson, I., Priddey, R. S., Isaak, K. G., \& McMahon, R. G. 2004, MNRAS, 351, L29

Rodighiero, G., Lari, C., Pozzi, F., et al. 2006, MNRAS, 371, 1891

Saracco, P., Longhetti, M., Severgnini, P., et al. 2005, MNRAS, 357, L40

Somerville, R. S., Primack, J. R., \& Faber, S. M. 2001, MNRAS, 320, 504

Somerville, R. S., Moustakas, L. A., Mobasher, B., et al. 2004, ApJ, 600, L135

Smail, I., Ivison, R. J., Blain, A. W., \& Kneib, J.-P. 2002, MNRAS, 331, 495

Smail, I., Chapman, S. C., Blain, A. W., \& Ivison, R. J. 2004, ApJ, 616, 71

Stanway, E. R., Bunker, A. J., \& McMahon, R. G. 2003, MNRAS, 342, 439

Stark, D. P., \& Ellis, R. S. 2006, New Astron. Rev., 50, 46

Steidel, C. C., Adelberger, K. L., Giavalisco, M., Dickinson, M., \& Pettini, M. 1999, ApJ, 519, 1

Steidel, C. C., Adelberger, K. L., Shapley, A. E., et al. 2003, ApJ, 592, 728

Stern, D., Chary, R.-R., Eisenhardt, P. R. M., \& Moustakas, L. A. 2006, AJ, 132, 1405

van Dokkum, P. G., \& Ellis, R. S. 2003, ApJ, 592, L53

Yan, H., Dickinson, M., Eisenhardt, P. R. M., et al. 2004, ApJ, 616, 63

Yan, H., Dickinson, M., Stern, D., et al. 2006, New Astron. Rev., 50, 127

Walter, F., Carilli, C., Bertoldi, F., et al. 2004, ApJ, 615, L17

Webb, T. M. A., van Dokkum, P., Egami, E., et al. 2006, ApJ, 636, L17

Zheng, W., Mikles, V. J., Mainieri, V., et al. 2004, ApJS, 155, 73 


\section{Online Material}


G. Rodighiero et al.: High redshift massive galaxies, Online Material $p 2$
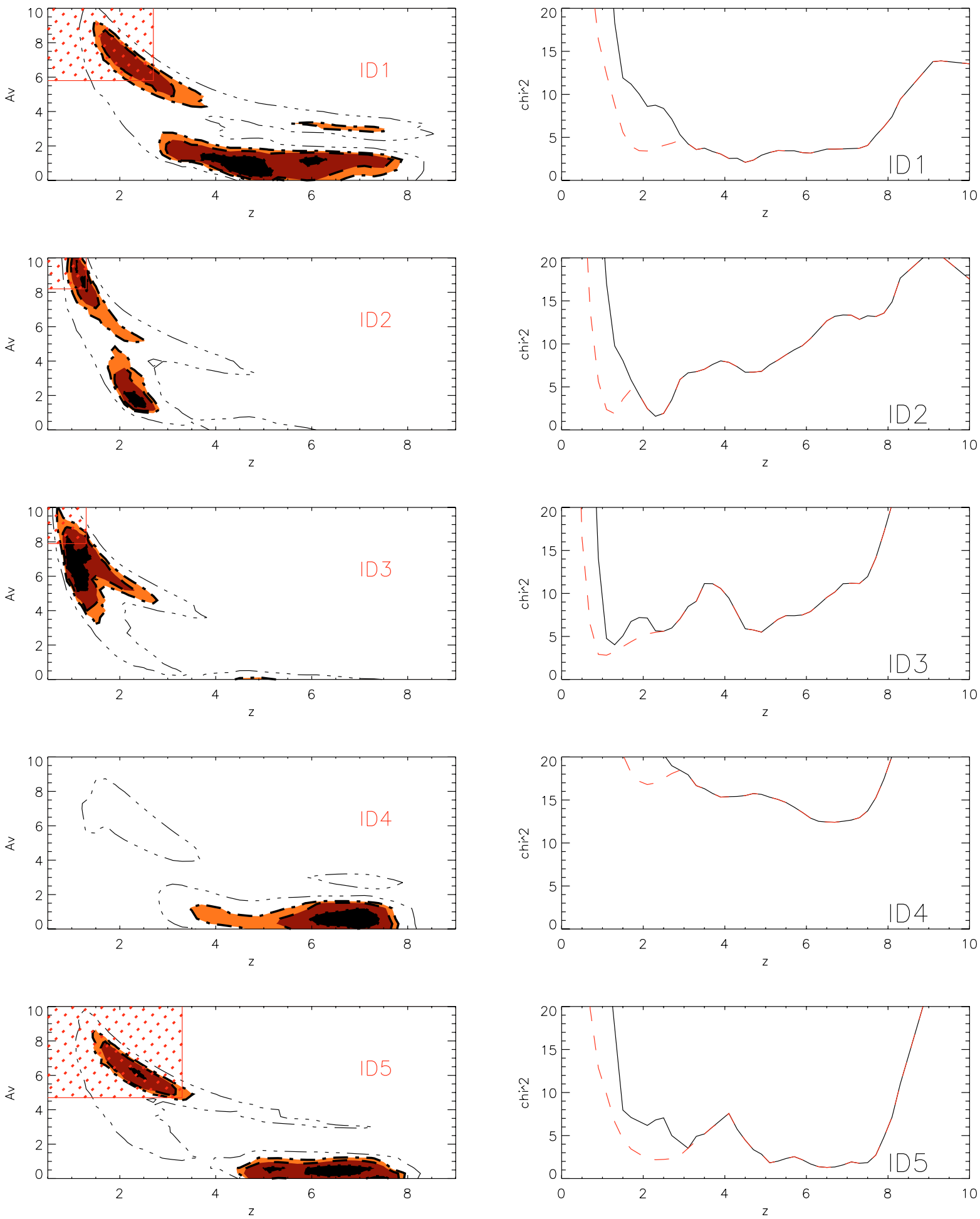

Fig. 8. Left panel: confidence levels for the photometric redshift $z$ and extinction $A_{\mathrm{V}}$ derived from a $\chi^{2}$ analysis for each source, for the $\mathrm{BC} 03$ model. Dotted, dashed, dot-dashed, and three-dot-dashed curves respectively mark the $68 \%, 90 \%, 95 \%$, and $99.99 \%$ confidence levels of the $\chi^{2}$ statistics. For each source, the red shaded area shows regions of the parameter space that appear to be disfavoured by our analysis in Sect. 4.1 taking the $24 \mu \mathrm{m}$ constraint into account. Right panel: the value of the $\chi^{2}$ a function of redshift. Different curves show the result of using different extinction ranges in the SED fitting procedure with Hyperz (solid curve: $A_{\mathrm{V}}<6$, red dashed curve: $A_{\mathrm{V}}<10$ ). 
G. Rodighiero et al.: High redshift massive galaxies, Online Material p 3
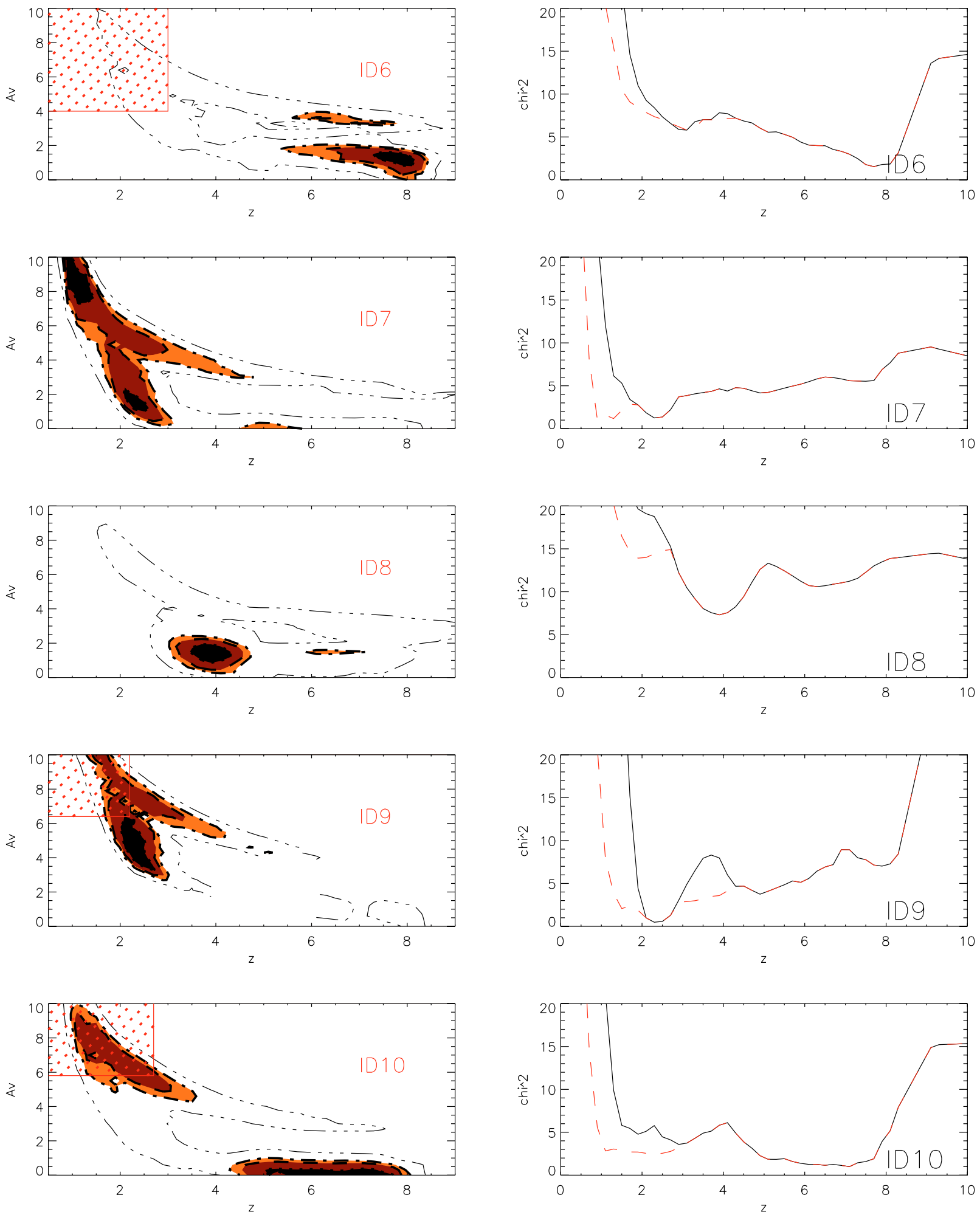

Fig. 8. continued. 
G. Rodighiero et al.: High redshift massive galaxies, Online Material p 4
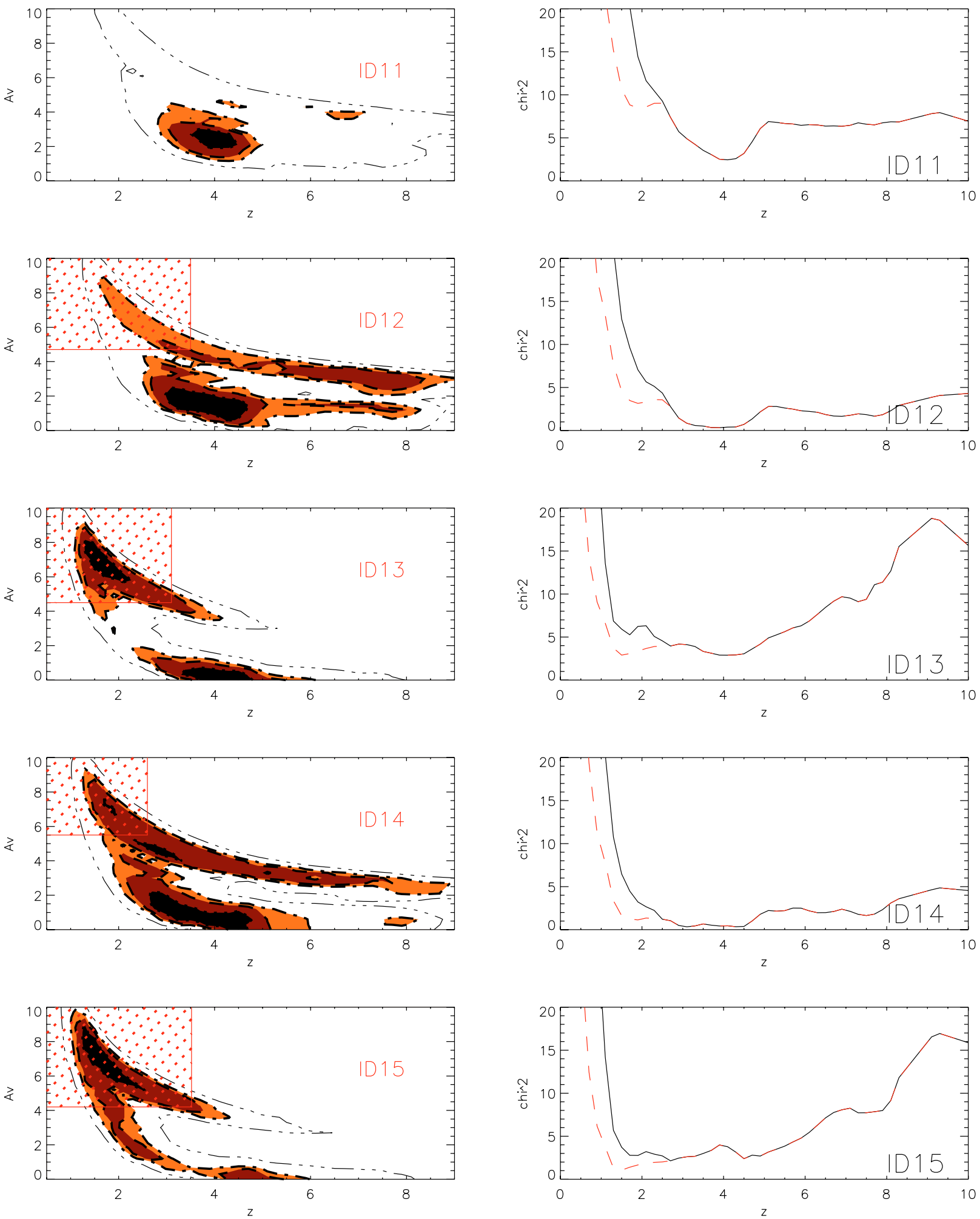

Fig. 8. continued. 
G. Rodighiero et al.: High redshift massive galaxies, Online Material p 5
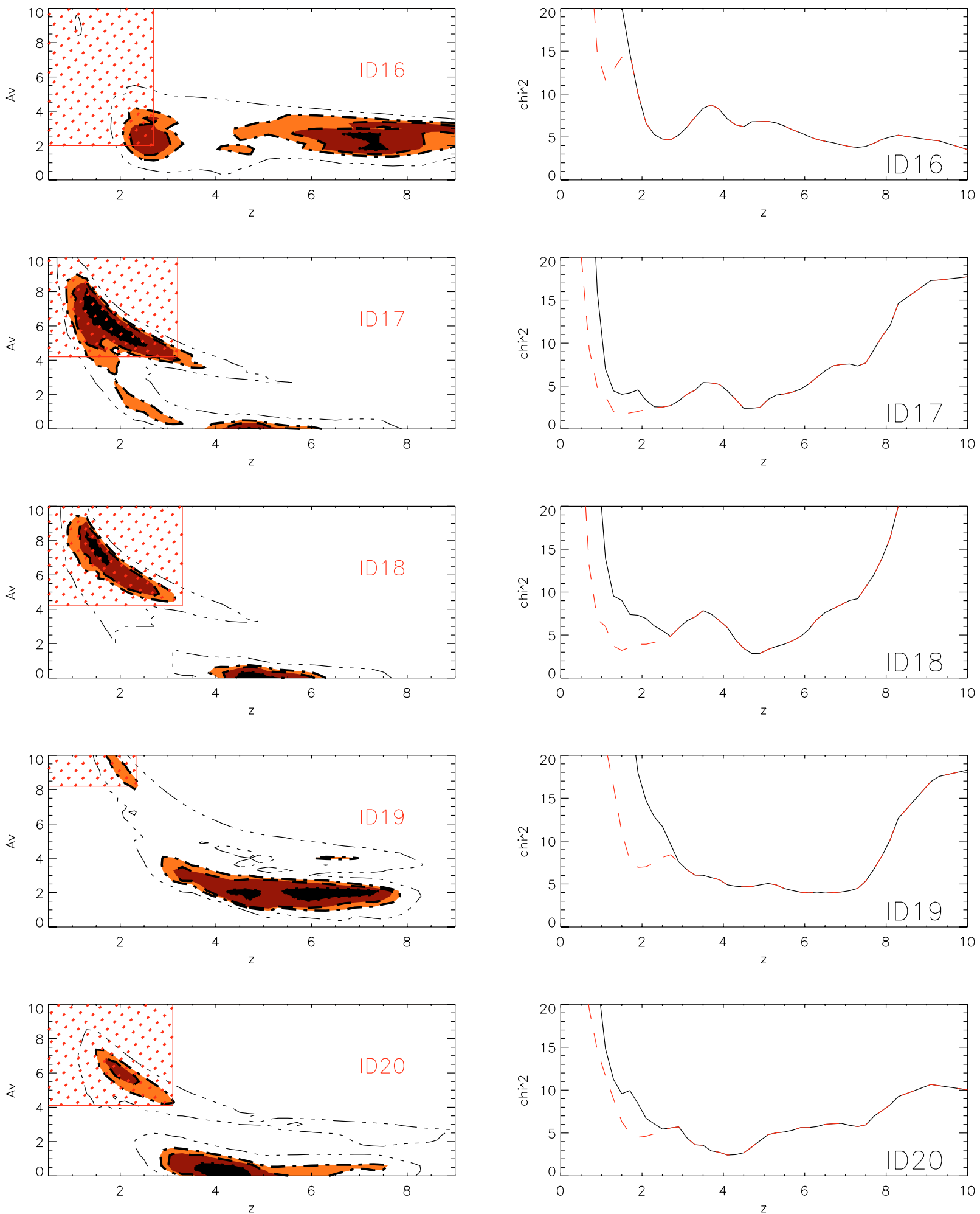

Fig. 8. continued. 
G. Rodighiero et al.: High redshift massive galaxies, Online Material $p 6$

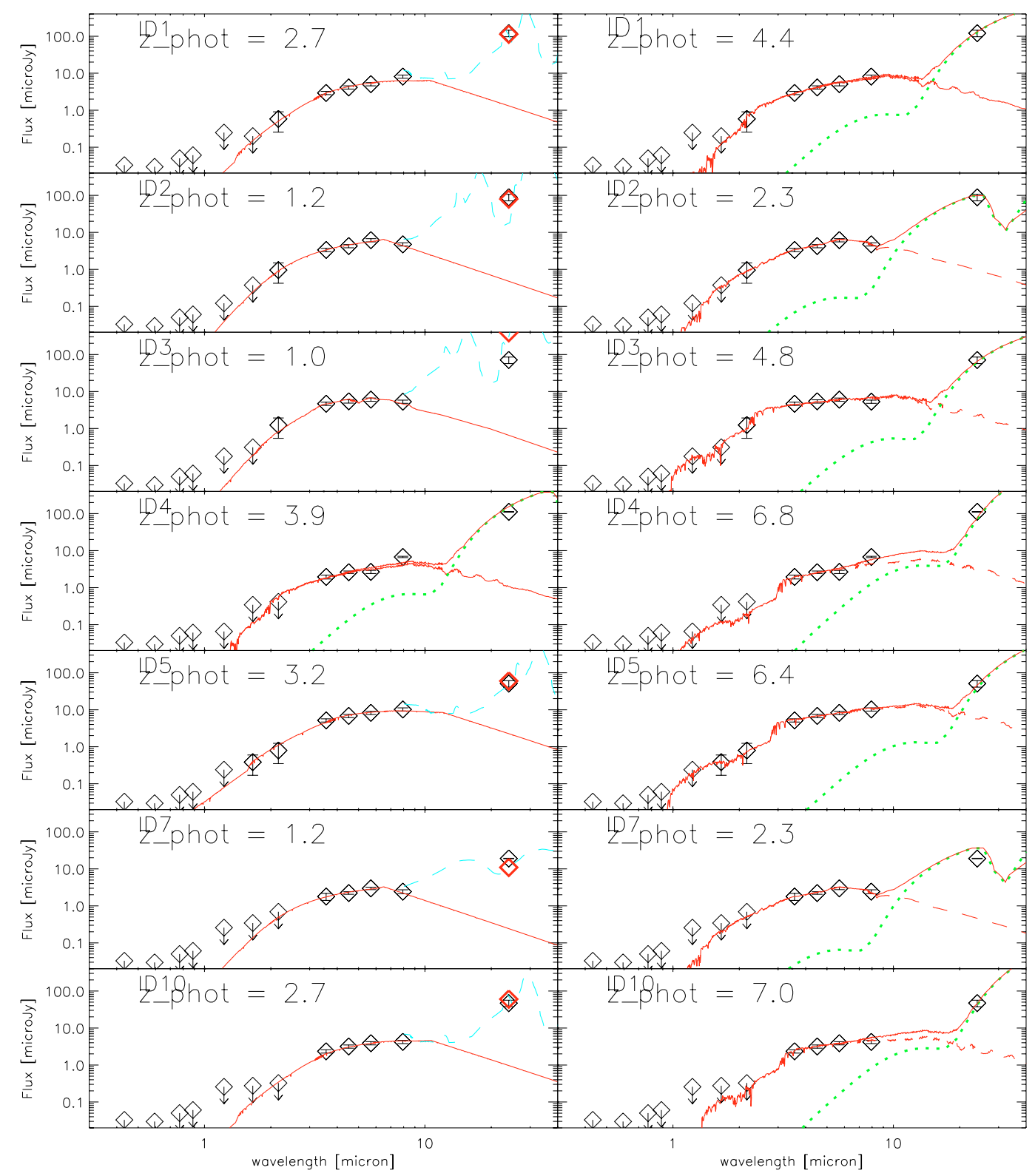

Fig. 9. Best-fit models for the sample sources with a bimodal solution. In the left panel we report the low-redshift solution. The observed SED of each source (open diamond) is shown with the corresponding low-redshift best-fit solution (solid red line) indicated by Fig. 8 and Table 3 for the stellar component (up to $8 \mu \mathrm{m}$ ). The IR starburst template that better reproduces the $S(24 \mu \mathrm{m}) / S(8 \mu \mathrm{m})$ flux ratio is reported as a dashed cyan line. The IR spectra have been normalized to match the $24 \mu \mathrm{m}$ measurements. In the right panel we show the observed SEDs with the corresponding best-fit high- $z$ solutions (dashed red lines, sol. II in Table 3) In this case, the IR part of the spectra have been reproduced with the spectral template of a dusty torus representing the emission of a type-2 AGN (green dotted lines, Fritz et al. 2006 model). The IR spectra have been normalized to match the $24 \mu \mathrm{m}$ measurements. The solid red lines correspond to the sum of the different galaxy components. 
G. Rodighiero et al.: High redshift massive galaxies, Online Material $p 7$

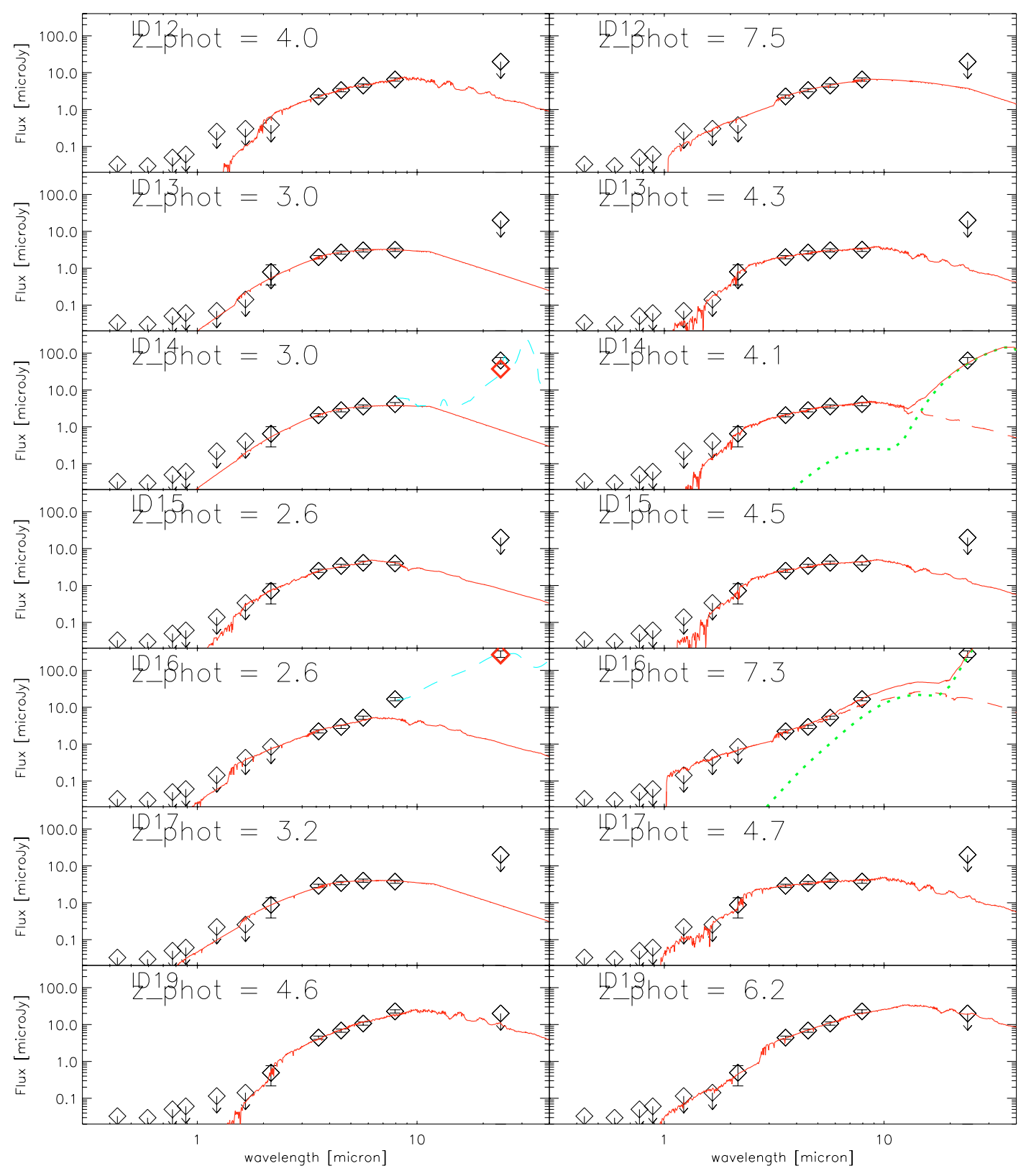

Fig. 9. continued. 
G. Rodighiero et al.: High redshift massive galaxies, Online Material $p 8$
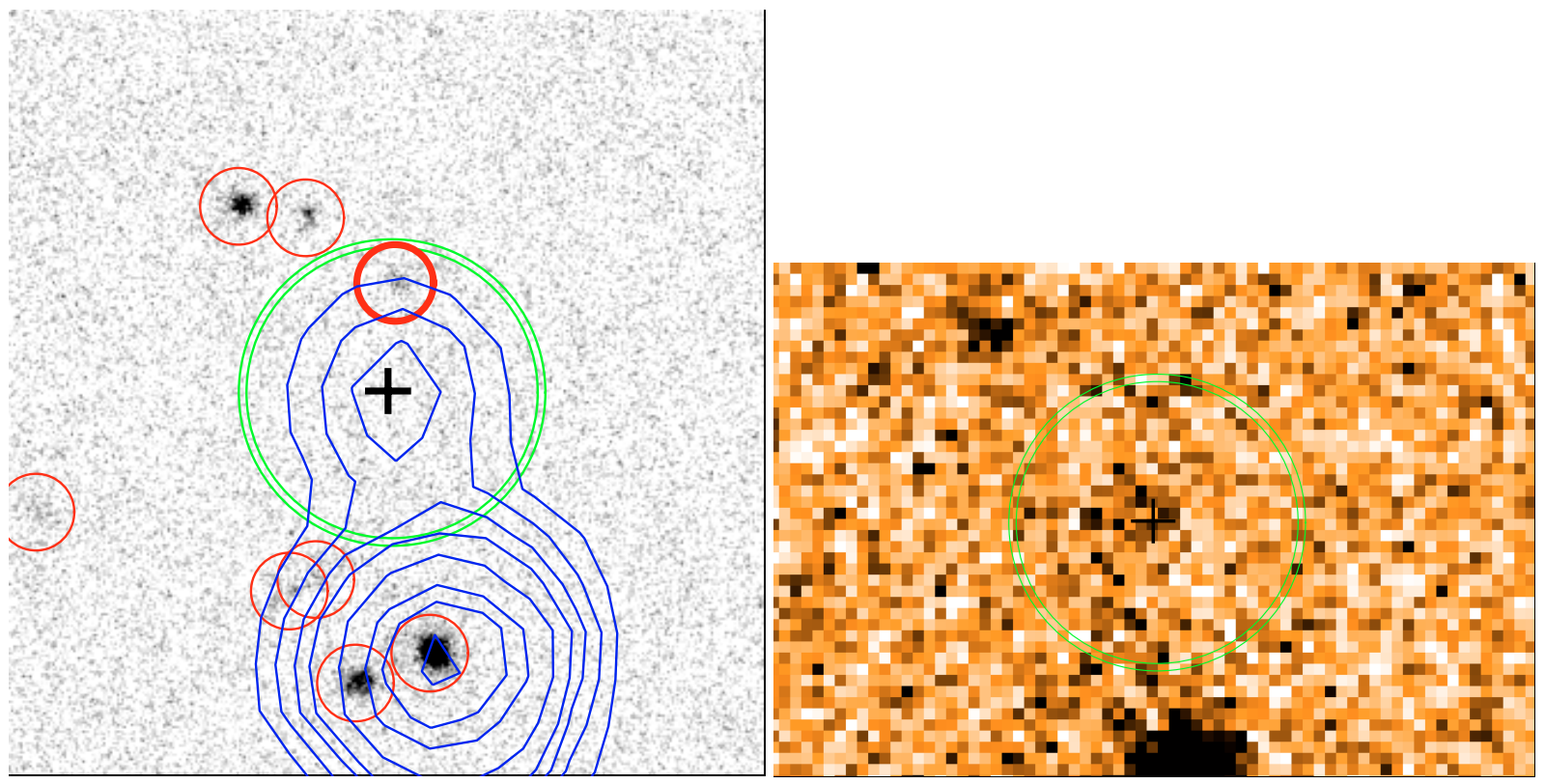

Fig. 13. Identification of source \#6. Left panel: the cutout shows a $10^{\prime \prime} \times 10^{\prime \prime}$ negative map of the HST $z$-band. The black cross marks the centroid of the $3.6 \mu$ m detected source, while the corresponding IRAC contours are shown in blue $(2,3,4,5,6,7,10,12,15 \sigma)$. The green circle ( $2^{\prime \prime}$ radius) represents the position of the X-ray source as reported by Alexander et al. (AID \#232). The red circles (1" diameter) indicate the optical sources detected in the field. The thicker red source is the optical counterpart for the X-ray source reported by Zheng et al. (2004) and Mainieri et al. (2005). Right panel: a $10^{\prime \prime} \times 7^{\prime \prime}$ map of the ISAAC $K$-band, with overlaid the circle indicating the position of the X-ray source ( $2^{\prime \prime}$ radius). There is a significant $2.2 \mu \mathrm{m}$ signal in correspondence with the IRAC centroid, excluding the identification with other sources in the optical map of the left panel. 
G. Rodighiero et al.: High redshift massive galaxies, Online Material $p 9$

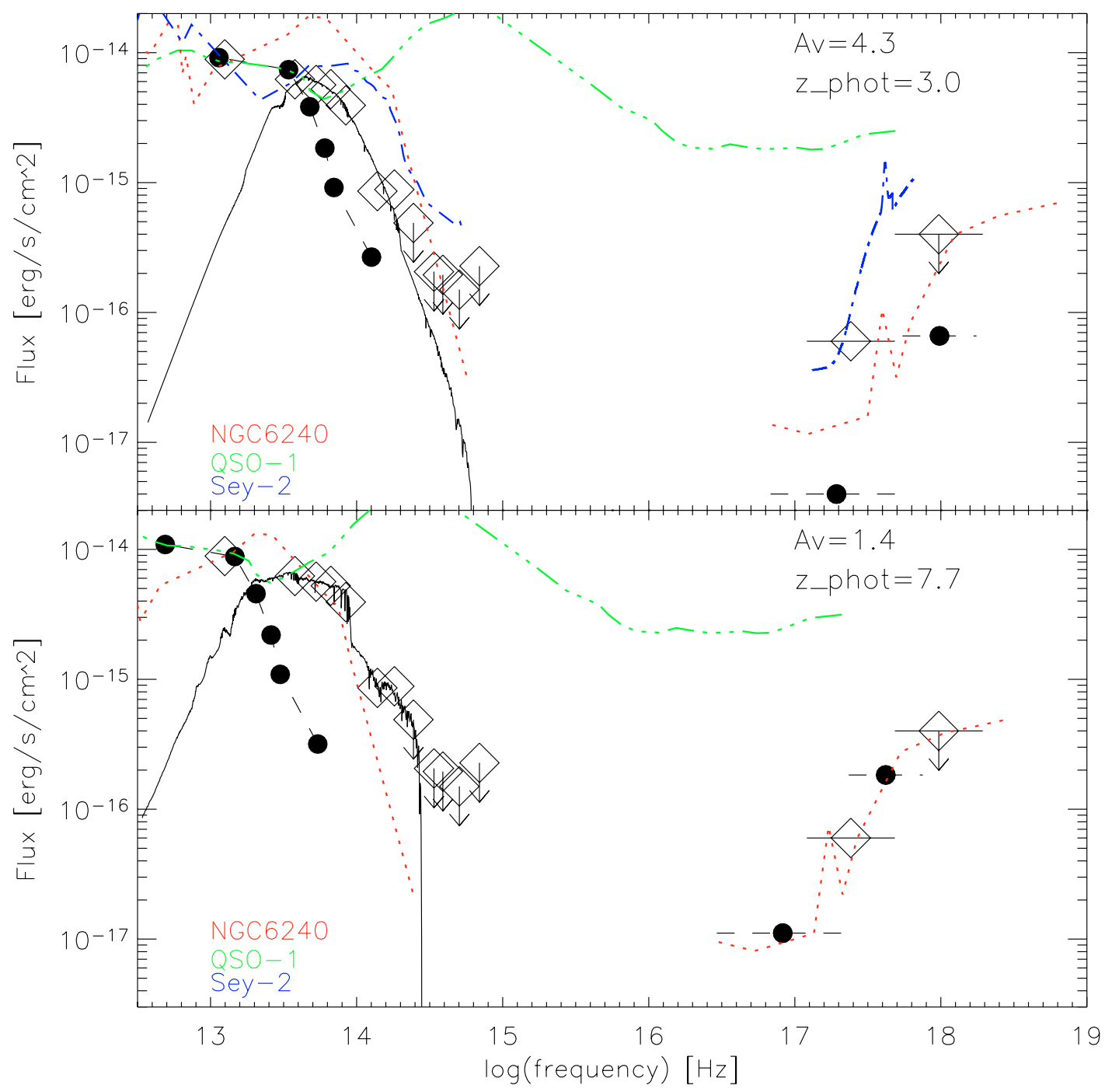

Fig. 14. The two main possible solutions for object ID\#6. In the lower panel we consider the primary solution at $z \sim 7.7$, while in the upper panel we show a secondary solution at $z \sim 3.0$ for comparison. The stellar component of the source (solid black lines) in both cases corresponds to the solution already explored in Sect. 4.1 and in Figs. 9-10. We compare the observations with various prototype templates: a type-1 QSO (three dots-dashed green lines, Elvis et al. 1994), the classic type-2 QSO NGC6240 (dotted red lines, Hasinger et al. 2001), and a typical Seyfert 2 (dot-dashed blue line). The models have been redshifted at the corresponding photometric redshifts and normalized to fit the observed $24 \mu \mathrm{m}$ flux. We also compared the two solutions with the recently discovered most luminous Compton-thick AGN at $z \sim 2.5$ (SWIRE J104409.95+585224.8, Polletta et al. 2006). The SED of this object (redshifted and normalized to the $24 \mu \mathrm{m}$ flux) is represented by the filled black circles. 
G. Rodighiero et al.: High redshift massive galaxies, Online Material p 10
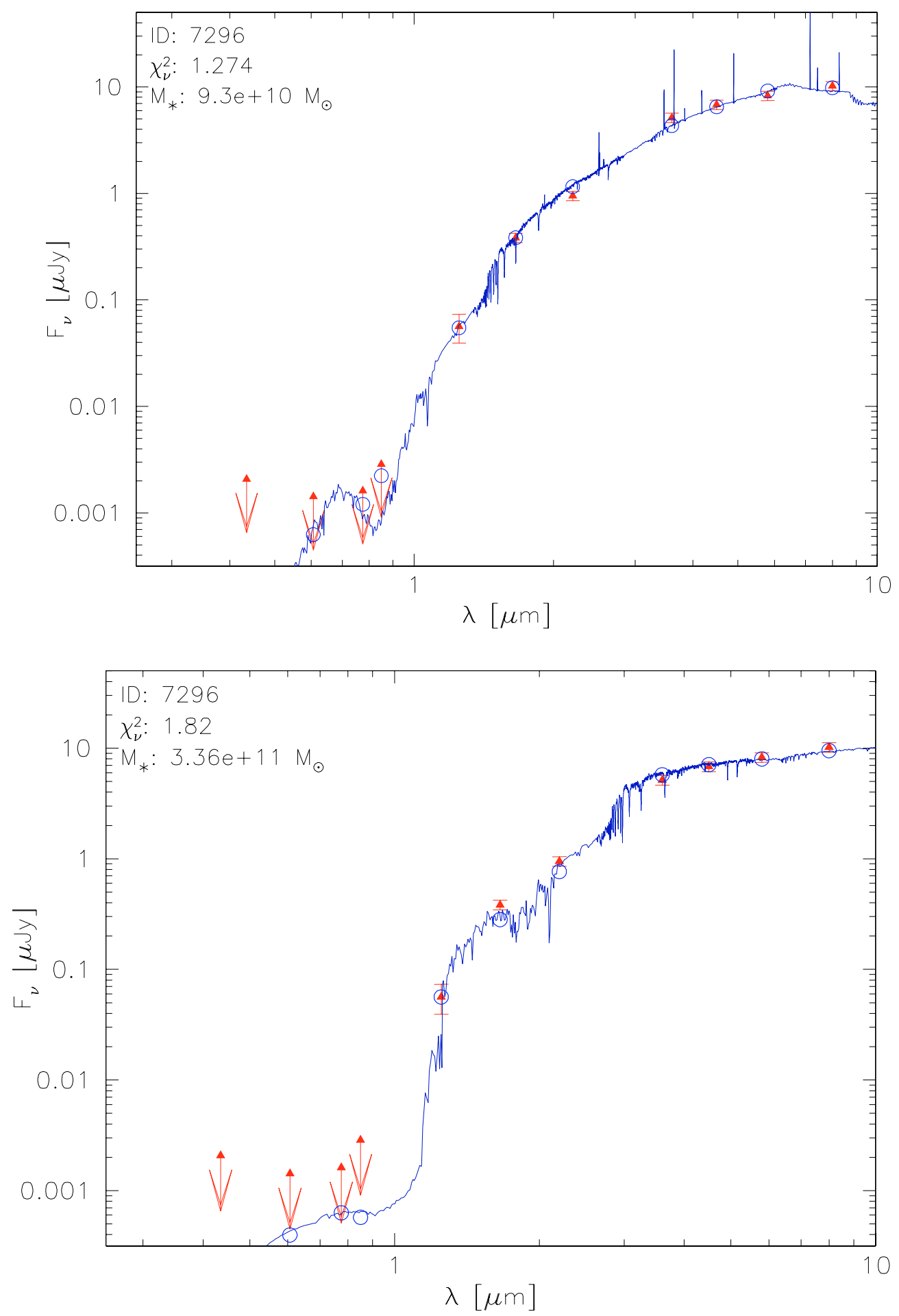

Fig. 15. SED analysis for source \#5 (the Mobasher et al. source) based on a detailed photometric model. The upper panel corresponds to the $z=2.82$ solution, the lower panel to the $z=6.5$ solution. The observed photometric data are marked as red-triangles. The best $-f i t$ model is represented with the blue lines. The convolution of the model in the various photometric bands is shown as open blue circles. Note that in this case we used Mobasher's photometry based on the UDF, in order to directly compare their results with our modellistic predictions. 
G. Rodighiero et al.: High redshift massive galaxies, Online Material p 11

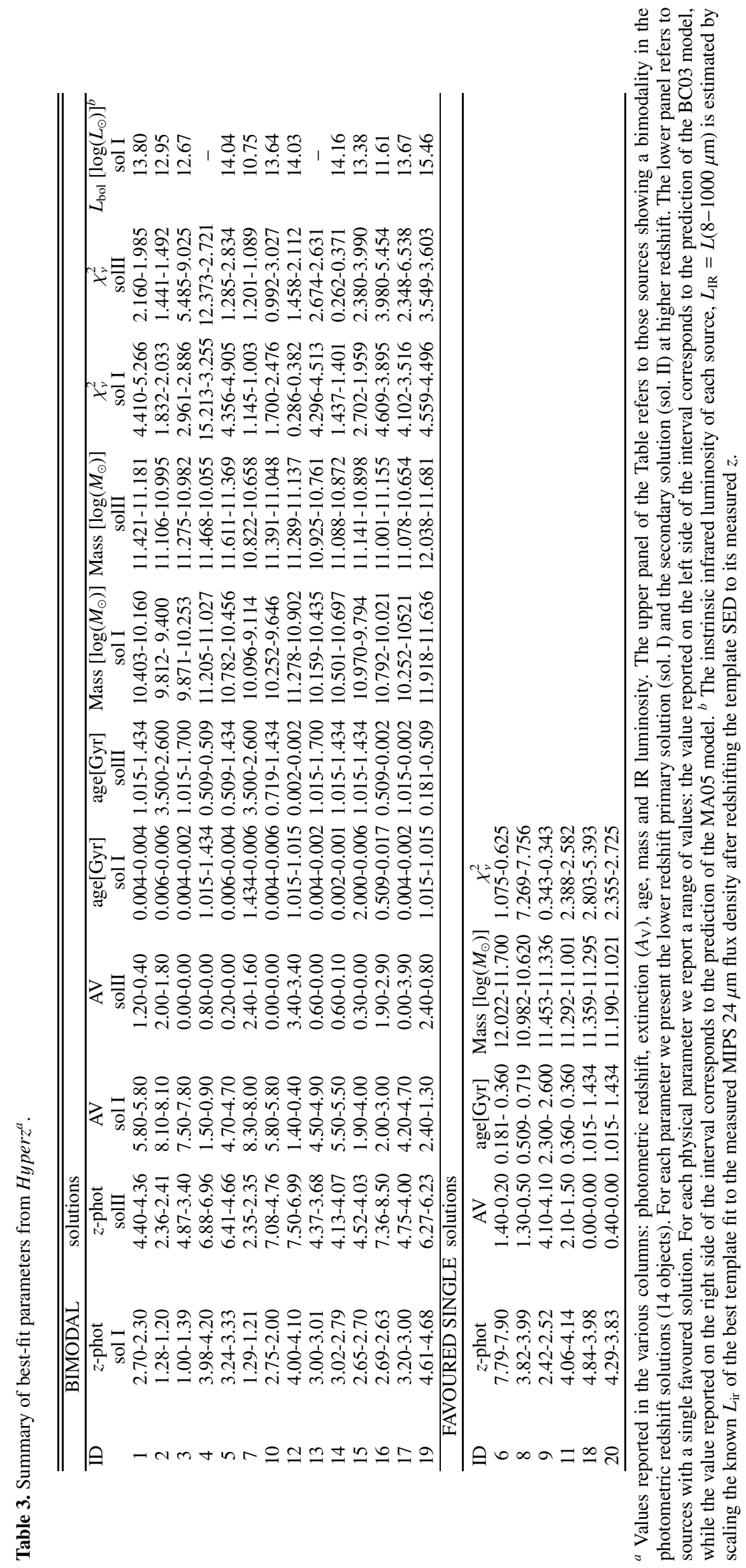

